\title{
Intermittent Behaviors in Coupled Piecewise Expanding Map Lattices
}

\author{
Tiexiang $\mathrm{Li}^{1,2}$, Wen-wei $\mathrm{Lin}^{3}$, Yiqian Wang ${ }^{4, *}$ \\ and Shing-Tung Yau ${ }^{5}$ \\ ${ }^{1}$ Department of Mathematics, Southeast Univeristy, Nanjing, Jiangsu 211189, China \\ 2 Nanjing Center for Applied Mathematics, Nanjing, Jiangsu 211135, China \\ ${ }^{3}$ Department of Mathematics, National Chiao Tung University, Hsinchu, 300, \\ Taiwan \\ 4 Department of Mathematics, Nanjing University, Nanjing, Jiangsu 210093, China \\ ${ }^{5}$ Department of Mathematics, Harvard University, Boston, USA
}

Received 4 May 2020; Accepted (in revised version) 8 September 2020

$$
\begin{aligned}
& \text { Abstract. In this paper, we propose a new method to study intermittent behaviors of } \\
& \text { coupled piecewise-expanding map lattices. We show that the successive transition be- } \\
& \text { tween ordered and disordered phases occurs for almost every orbit when the coupling } \\
& \text { is small. That is, } \\
& \qquad \liminf _{n \rightarrow \infty} \sum_{1 \leq i, j \leq m}\left|x_{i}(n)-x_{j}(n)\right|=0, \\
& \qquad \limsup _{n \rightarrow \infty} \sum_{1 \leq i, j \leq m}\left|x_{i}(n)-x_{j}(n)\right| \geq c_{0}>0,
\end{aligned}
$$
coupled piecewise-expanding map lattices. We show that the successive transition between ordered and disordered phases occurs for almost every orbit when the coupling

where $x_{i}(n)$ correspond to the coordinates of $m$ nodes at the iterative step $n$. Moreover, when the uncoupled system is generated by the tent map and the lattice consists of two nodes, we prove a phase transition occurs between synchronization and intermittent behaviors. That is,

$$
\lim _{n \rightarrow \infty}\left|x_{1}(n)-x_{2}(n)\right|=0 \text { for } \quad\left|c-\frac{1}{2}\right|<\frac{1}{4}
$$

and intermittent behaviors occur for $\left|c-\frac{1}{2}\right|>\frac{1}{4}$, where $0 \leq c \leq 1$ is the coupling.

Key Words: Synchronization, pseudo synchronization, phase transition, Coupled map Lattices, piecewise expanding map.

AMS Subject Classifications: 37D20, 37A25

*Corresponding author. Email addresses: txli@seu.edu.cn (T. X. Li), wwlin@math.nctu.edu.tw (W. W. Lin), yiqianw@nju.edu.cn (Y. Q. Wang), yau@math.harvard.edu (S. T. Yau) 


\section{Introduction}

In this paper, we study the intermittent dynamical behavior of coupled piecewiseexpanding map lattices. Let $f:[0,1] \rightarrow[0,1]$ be a piecewise expanding map, $I$ be the $m \times m$ identity matrix and $A$ be an $m \times m$ symmetric matrix satisfying $A \mathbf{e}=0$, where $\mathbf{e}=[1, \cdots, 1]^{\top}$. Consider the dynamical system defined by a coupled map lattice:

$$
T: \quad \mathbf{x}(n+1)=(I+c A) \mathbf{f}(\mathbf{x}(n)),
$$

where $c$ is the coupling coefficient, $\mathbf{x}(n)=\left[x_{1}(n), \cdots, x_{m}(n)\right]^{\top} \in[0,1]^{m}$ for $n \in \mathbb{N} \cup\{0\}$ and $\mathbf{f}(\mathbf{x}(n))=\left[f\left(x_{1}(n)\right), \cdots, f\left(x_{m}(n)\right)\right]^{\top}$. In case of no confusion, we also use bold letters $\mathbf{x}$ or $\mathbf{p}=\left(x_{1}, \cdots, x_{m}\right)$ to denote points in $[0,1]^{m}$.

Because of $A \mathbf{e}=0$, it can be easily seen that the diagonal $D_{\text {syn }}=\left\{\left(x_{1}, \cdots, x_{m}\right) \in\right.$ $\left.[0,1]^{m} \mid x_{1}=\cdots=x_{m}\right\}$ is an invariant set for synchronized points of $T$. An interesting question on the dynamical behavior of the coupled map lattice (1.1) can be raised as whether $D_{\text {syn }}$ is a global attractor, or equivalently, whether synchronization occurs for (1.1). There have been plenty of results on the study of synchronization when $f$ generates a chaotic dynamical system. Common examples include the tent maps and the Logistic maps, one can see $[2,20,27]$ and references therein. It has been shown in these results that chaotic synchronization can occur only if $c$ is far from zero. That is, chaotic synchronization can not occur for small coupling strength.

However, a more complicated phenomenon has been found by numerical simulations when $c$ is out of the synchronized region. Roughly speaking, it is found that a typical orbit can enter into and exits slowly from an arbitrarily small neighborhood of $D_{\text {syn }}$ for infinite times. In other word, the successive transition between being close to the diagonal and being far from the diagonal can happen. We call this phenomenon as pseudo-synchronization.

The pseudo-synchronization is closely related to the clustering phenomenon in global coupled map lattices by Kaneko et al. [4,9-14]. In numerical experiments, it showed that when (1.1) is a globally coupling system with large $m$, elements differentiate into some clusters, and elements in each cluster oscillate synchronously, while the behaviors in different clusters are various. Moreover, the differentiation by clustering is a temporal behavior in nature [14]. One can easily see that the pseudo-synchronization is a special case of the temporal clustering. In fact, the temporal clustering is also found in all systems of (1.1) with small $c$. Similar behaviors were also widely explored in weakly coupled continuous-time chaotic systems. For example, the successive transition between bursting and spiking was discovered in the study of epilepsy, see $[5,6]$ and references therein. More related results are shown in $[3,7,24,25]$ and references therein. To provide a mathematical proof for the mechanism of pseudo-synchronization for coupled map lattices is one of motivations of this paper.

On the other hand, there are a series of mathematical results on dynamical behaviors of weakly-coupled map lattices. In [15], Keller showed that the existence of unique 
absolutely continuous invariant measure for weakly-coupled tent maps. Keller and Liverani [18] proved the existence of the unique SRB measure for a wide range of multidimensional weakly coupled map lattices. They also showed the exponential decay of correlations in time and space in some one-dimensional lattices of weakly coupled piecewise expanding interval maps [17]. Furthermore, they provide some exact formulae to describe the escape rates with respect to the infinitesimal neighborhood of the synchronization manifold for the coupled systems [19]. More further results can be found in $[1,8,16,21,22,26]$ and references therein. Another motivation of this paper is therefore to provide a precise description on the set of the couplings corresponding to intermittent behaviors and escape rates with respect to the synchronization manifold of coupled map lattices.

In this paper, we will prove the occurrence of successive transitions for almost every point in the sense of Lebesgue measure for the following coupled map lattices, where $f$ is the tent map or its perturbation.

In (1.1), when $m=2$, we have the following coupled map lattice:

$$
T:\left\{\begin{array}{l}
x_{1}(n+1)=(1-c) f\left(x_{1}(n)\right)+c f\left(x_{2}(n)\right), \\
x_{2}(n+1)=c f\left(x_{1}(n)\right)+(1-c) f\left(x_{2}(n)\right) .
\end{array}\right.
$$

Let $\operatorname{dist}(A, B)$ denote the distance between two points/sets $A$ and $B$. Numerical simulation shows that when $f(x)$ is piecewise-expanding and close to the standard tent map, and $c$ is smaller than some $c_{+}>0$, the pseudo-synchronization occurs for the system (1.2). That is,

$$
\begin{aligned}
& \liminf _{n \rightarrow \infty} \operatorname{dist}\left(\mathbf{x}(n), D_{\text {syn }}\right)=0, \\
& \limsup _{n \rightarrow \infty} \operatorname{dist}\left(\mathbf{x}(n), D_{\text {syn }}\right) \geq \gamma_{0}>0,
\end{aligned}
$$

for some $\gamma_{0}>0$. In other word, the successive transition between the order phase (close to $D_{\text {syn }}$ ) and the disorder phase (far from $D_{\text {syn }}$ ) occurs for almost every orbit. Similar behaviors are also found for the multi-node cases.

Obviously, (1.3a) and (1.3b) are equivalent to the following equations, respectively,

$$
\begin{aligned}
& \liminf _{n \rightarrow \infty}\left|x_{1}(n)-x_{2}(n)\right|=0, \\
& \limsup _{n \rightarrow \infty}\left|x_{1}(n)-x_{2}(n)\right| \geq \gamma_{0}>0 .
\end{aligned}
$$

In this paper, we will provide such a series of mathematical proofs for Theorem 1.1Theorem 1.4 as follows.

Theorem 1.1. Consider the system (1.2) with $f(x)=1-2\left|x-\frac{1}{2}\right|, x \in[0,1]$ being the standard tent map. Then for all $0 \leq c<\frac{1}{4}$ (or $\frac{3}{4}<c \leq 1$ ), there exists a constant $\gamma_{0}>0$ such that for 
almost every initial point $\left(x_{1}(0), x_{2}(0)\right) \in[0,1]^{2},(1.3 \mathrm{a})$ and $(1.3 \mathrm{~b})$ hold true. Moreover, the synchronization occurs for each $\frac{1}{4}<c<\frac{3}{4}\left(c=\frac{1}{4}\right.$ or $\left.\frac{3}{4}\right)$, that is,

$$
\lim _{n \rightarrow \infty} \operatorname{dist}\left(\mathbf{x}(n), D_{\text {syn }}\right)=0
$$

for (almost) every initial data.

Remark 1.1. Thus we conclude that $c=\frac{1}{4}$ is the bifurcation point between synchronization described by (1.4) and intermittent behavior described by (1.3a)-(1.3b). Hopefully it can be generalized to the situation with any $m$ nodes as well as with a non-piecewiselinear perturbation on $f$, which will be studied in our future work.

For non-piecewise-linear $f$, the situation is more difficult, for which we will provide a method different from the one for Theorem 1.1. To show the idea for non-piecewiselinear situation, we would rather first prove the following result weaker than Theorem 1.1.

Theorem 1.2. Consider the system (1.2) with $f(x)=1-2\left|x-\frac{1}{2}\right|, x \in[0,1]$ being the standard tent map. There exists $0<c_{+} \leq \frac{1}{4}$ such that if the coupling coefficient $0 \leq c<c_{+}$, then there exists a constant $\gamma_{0}>0$ such that for almost every initial point $\left(x_{1}(0), x_{2}(0)\right) \in[0,1]^{2},(1.3 \mathrm{a})$ and $(1.3 \mathrm{~b})$ hold true.

Remark 1.2. The function $f(x)$ in the system (1.2) need not be the standard tent map. We can prove that there exist constants $c_{+}, \alpha_{+}>0$ such that the same conclusion as in Theorem 1.2 holds true for the general tent map

$$
f(x)= \begin{cases}\left(2-\alpha_{1}\right) x, & 0<x \leq \frac{1}{2}-\alpha_{2}, \\ \frac{1-2 \alpha_{2}}{1+2 \alpha_{2}}\left(2-\alpha_{1}\right)(1-x), & \frac{1}{2}-\alpha_{2}<x \leq 1,\end{cases}
$$

with $|c| \leq c_{+}$and $\left|\alpha_{i}\right|<\alpha_{+}, i=1,2$. Moreover, the result can be extended to the general piecewise linear continuous function $f$ with slopes being large enough.

The conclusion in Theorem 1.2 can be generalized to the case that $f$ is piecewise expanding. More precisely, we will prove that

Theorem 1.3. Let $f_{0}(x)=1-s\left|x-\frac{1}{2}\right|, x \in[0,1]$, where $s=2-s_{0}$ with $0 \leq s_{0}<1$. and $g(x)$ be a $\mathrm{C}^{2}$-smooth function on $x \in[0,1]$ such that $f(x)=f_{0}(x)+g(x) \in[0,1]$ for each $x$. Then there exist three small constants $c_{+}, s_{0+}, \eta>0$ such that if $0 \leq c<c_{+}, s_{0} \leq s_{0+}$ and $\|g\|_{e^{2}}<\eta$, there exists a constant $\gamma_{0}>0$ such that for almost every initial point $\left(x_{1}(0), x_{2}(0)\right) \in[0,1]^{2}$, (1.3a) and (1.3b) hold true for the system (1.2).

Theorem 1.2 can also be extended to the multi-node case. 
Theorem 1.4. Let $f$ be the standard tent map and consider the coupled tent map lattices (1.1) with $A$ being $m \times m$ symmetric matrix satisfying Ae $=0$. There exists $c_{+}>0$ such that if the coupling coefficient $0 \leq c<c_{+}$, then there exists a constant $\gamma_{0}>0$, such that for almost every initial point $\left(x_{1}(0), \cdots, x_{m}(0)\right) \in[0,1]^{m},(1.3 \mathrm{a})$ and $(1.3 \mathrm{~b})$ hold true.

Remark 1.3. Remark 1.2 is also applicable for Theorems 1.3 and 1.4.

Theorem 1.2 is a special case of Theorem 1.3 or 1.4 without regard to the fact we can obtain a larger $c_{+}$in Theorem 1.2 than in other two theorems. However, we will still give the proof of Theorem 1.2 first, since it is helpful for readers to understand the key idea of the proof as well as more complicated cases considered in Theorem 1.3.

Remark 1.4. In principle, similar results as in Theorems 1.3 and 1.4 can be extracted from [15] by Keller and [22] by Liverani with tranditional functional analysis methods. However, our geometric method is completely new. More importantly, the parameters in theorem 1.1 based on our new method is not necessary to be small. In fact, we obtained a phase transition result. In contract, the parameters in [15] and [22] are required small and it seems difficult to determine explicitly whether a parameter is sufficiently small. From the view point of application, the result in Theorem 1.1 is much better than related ones obtained by the functional analysis method in $[15,22]$. Potentially, a precise description on the set of the couplings corresponding to intermittent behaviors for general situations can be provided by our new method.

The remaining part of this paper is organized as follows. In Section 2, we give a key iteration lemma as the base for the main proof. In Sections 3 and 4, we prove (1.3a) (the ordered part) and (1.3b) (the disordered part) of Theorem 1.2. The proof of Theorem 1.3 is given in Section 5. In the next section, we will prove Theorem 1.4. The proof of Theorem 1.1 will be given in the last section.

\section{The basic idea and the key lemma}

In this section, we will describe our intuition for the proof. From the observation, we then provide a key iteration lemma, which is the base for the main proof.

Roughly speaking, for any set $S$ with a small measure in some sense, from the local expansion of the map $T$, we observe that the measure of $T^{j}(S)$ will become large enough for some large $j$ such that $T^{j}(S) \cap D_{\text {syn }} \neq \varnothing$. If $T^{i}(S)$ also satisfies some "good" property for $i=0,1, \cdots, j$ (say, $T^{i}(S)$ is a segment or convex region), then we can show that for any neighborhood of the diagonal $D_{\text {syn }}$ there is a constant $m_{0}>0$ such that there exists a subset $S_{0}$ of $S$ satisfying that (i) for each point $\mathbf{p} \in S_{0}, T^{j}(\mathbf{p})$ is in the neighborhood of $D_{\text {syn }}$; (ii) $\mathrm{M}\left(S_{0}\right) \geq m_{0} \mathrm{M}(S)$, where $\mathrm{M}(\cdot)$ denotes the Lebesgue measure or a domain of the length of a simple curve. Then (i) and (ii) will imply (1.3a) holds true for a set of full measure.

However, since $T$ is not one to one and thus is not globally expanding, the actual picture is much more complicated than the one described above. In fact, since $T$ is not 
one-to-one, usually the "good" property of a set $S$ is not preserved by its image $T(S)$. Without this property, it is impossible to obtain (ii). On the other hand, let $[0,1]^{m}=\cup D_{J}$, where $D_{J}$ are $2^{m}$ small hypercubes of $[0,1]^{m}$ divided by the planes $x_{i}=\frac{1}{2}, 1 \leq i \leq m$. From the definition of $f$, one can see that $T: D_{J} \rightarrow[0,1]^{m}$ is one to one for each $J$. Moreover, $T\left(D_{J} \cap S\right)$ will keep the "good" property of $S$. For this reason, we have to divide $S$ into $S \cap D_{J}$, and consider the iterations of $T$ on each of them individually.

For convenience, we say $S$ has $i_{1}$ components if there are $i_{1}$ nonempty sets among all $S \cap D_{J}$. Furthermore, consider a set $D \subset[0,1]^{m}$. For any set $S$ with components $S_{1,1}, \ldots, S_{1, i_{1}}$, if there are exactly $\hat{i}_{1}$ components $S_{1,1_{j}}, j=1, \ldots, \hat{i}_{1} \leq i_{1}$ among them such that $S_{1,1} \cap D \neq \varnothing$ for each $1 \leq j \leq \hat{i}_{1}$, we say $S$ has $\hat{i}_{1}$ components in $D$. For the set $S$ stated above, suppose for each $1 \leq j \leq i_{1}, T\left(S_{1, j}\right)$ has $k_{1}(j)$ components, we say $T(S)$ has $\sum_{j=1}^{i_{1}} k_{1}(j)$ components. Similarly, suppose for each $1 \leq j \leq \hat{i}_{1}, T\left(S_{1,1_{j}}\right)$ has $\hat{k}_{1}(j)$ components in $D$, we say $T(S)$ has $\sum_{j=1}^{\hat{i}_{1}} \hat{k}_{1}(j)$ components in $D$. Inductively, suppose $T^{l}(S)$ has components $S_{l, 1}, \ldots, S_{l, i_{l}}$ and has components $S_{l, l_{j}}, j=1, \ldots, \hat{i}_{l} \leq i_{l}$ in $D$. Furthermore, for each $1 \leq j \leq i_{l}, T\left(S_{l, j}\right)$ has $k_{l}(j)$ components, and for $1 \leq j \leq \hat{i}_{l}$, $T\left(S_{l, l_{j}}\right)$ has $\hat{k}_{l}(j)$ components in $D$. We say that $T^{l+1}(S)$ has $\sum_{j=1}^{i_{l}} k_{l}(j)$ components and $\sum_{j=1}^{\hat{i}_{l}} \hat{k}_{l}(j)$ components in $D$, respectively. In the same way, we can give the definitions for $T^{-l}(S)$ (in $D$ ). Note that each component $\Omega$ of $T^{l}(S)$ corresponds a subset $\Omega_{0} \subset S$ such that $T^{l}: \Omega_{0} \rightarrow \Omega$ is a homeomorphism.

Obviously, the measure of each component of $S$ is usually strictly less than the measure of $S$. Moreover, images of each component may have more than one component. Thus we need to show that averagely the local expansion of the map will surpass the dividing action by $x_{i}=\frac{1}{2}, i=1, \cdots, m$ on a set so that the measures of the components will keep increasing as the iteration goes forward only if the intersection of the corresponding set and $D_{\text {syn }}$ is empty. More detailed, we have the key iteration lemma and its corollary as below.

Consider the coupled map lattice $T$ in (1.1) with $f(x)$ differential for $x \neq \frac{1}{2}$. Let $S \subset[0,1]^{m}$ be a simple curve or measurable set. We define

$$
E_{+}(c)= \begin{cases}\sup _{\mathbf{p} \in[0,1]^{m}}|\operatorname{det}(J T(\mathbf{p}, c))|=|\operatorname{det}(A(c))| v_{+}^{m} & \text { w.r.t. a measurable set } S, \\ \sup _{\mathbf{p} \in[0,1]^{m}}\|J T(\mathbf{p}, c)\| & \text { w.r.t. a simple curve } S,\end{cases}
$$

where $A(c) \equiv I+c A$ is the coupling matrix, $J T(\mathbf{p}, \cdot)$ be the Jacobian matrix of $T$ at $\mathbf{p}$ (if it exists) and $v_{+}=\sup _{x \neq \frac{1}{2}}\left|f^{\prime}(x)\right|$. Similarly, we define

$$
E_{-}(c)= \begin{cases}\inf _{\mathbf{p} \in[0,1]^{m}}|\operatorname{det}(J T(\mathbf{p}, c))|=|\operatorname{det}(A(c))| v_{-}^{m} & \text { w.r.t. a measurable set } S, \\ \inf _{\mathbf{p} \in[0,1]^{m}}\|J T(\mathbf{p}, c)\| & \text { w.r.t. a simple curve } S,\end{cases}
$$


where $v_{-}=\inf _{x \neq \frac{1}{2}}\left|f^{\prime}(x)\right|$. Obviously, for any simple curve or measurable set $S$ in some small cube $D_{J}$ of the phase space, we have

$$
E_{-}(c) \mathrm{M}(S) \leq \mathrm{M}(T(S)) \leq E_{+}(c) \mathrm{M}(S)
$$

For example, if $f$ is the tent map and $m=2$, then $E_{ \pm}(c)$ equals $4(1-2 c)$ for a measurable set and $E_{+}(c)=2, E_{-}(c)=2(1-2 c)$ for a curve.

For any real number $x$, we define $\lfloor x\rfloor=\max \{i$ is an integer $\mid i \leq x\}$. Let $D$ be a domain in the phase space $[0,1]^{m}$.

Definition 2.1. Let $\delta>0, m_{0}, a \in \mathbb{N}$. We say a simple curve or measurable set $S$ in $D$ is $\left(\delta, m_{0}, a\right)$-good if $S$ lies in some small cube of $[0,1]^{m}$ with $\mathrm{M}(S) \leq \delta \mathrm{M}(D)$ and for each $i \geq 0$ and each component $S_{1}$ of $T^{i}(S)$ with $\mathrm{M}\left(S_{1}\right) \leq \delta \mathrm{M}(D)$, it holds that $T^{m_{0}}\left(S_{1}\right)$ has at most a components.

Lemma 2.1 (Iteration Lemma). Assume $E_{+}(c) \geq E_{-}(c)>1$. Let $D$ be a domain in the phase space $[0,1]^{m}$. Suppose $0<\delta_{1}<1$ and $a<E_{-}^{m_{0}}(c)$ with $a, m_{0} \in \mathbb{N}$. Let

$$
\begin{aligned}
& 1<\mu<\left(1-\log _{E_{+}(c)} \frac{E_{-}(c)}{a^{1 / m_{0}}}\right)^{-1}, \\
& d=1-\left(1-\log _{E_{+}(c)} \frac{E_{-}(c)}{a^{1 / m_{0}}}\right) \mu>0, \\
& F(c)=a\left(\frac{E_{-}(c)}{a^{1 / m_{0}}}\right)^{1-\log _{E_{+}(c)} \delta_{1}} .
\end{aligned}
$$

Define

$$
N_{0}=\left\lfloor\log _{\mu}\left(d^{-1} \log _{2} F(c)\right)\right\rfloor .
$$

Suppose $N>N_{0}$ and for any $\left(\delta_{1}, m_{0}, a\right)$-good curve or measurable set $\Omega \subset D$ with $\mathrm{M}(\Omega) \in$ $\left[2^{-\mu^{N+1}} \mathrm{M}(D), 2^{-\mu^{N}} \mathrm{M}(D)\right]$, define

$$
k(N)=\left\lfloor-\log _{E_{+}(c)} \frac{\mathrm{M}(\Omega)}{\delta_{1} \mathrm{M}(D)}\right\rfloor .
$$

Then there exist some disjoint subcurves or measurable subsets $\Omega_{j} \subset \Omega, j=1, \cdots$, such that (i) for each $j \geq 1$ it holds that $T^{k(N)}\left(\Omega_{j}\right)$ is a component of $T^{k(N)}(\Omega)$ with $\mathrm{M}\left(T^{k(N)}\left(\Omega_{j}\right)\right)$ $\geq 2^{-\mu^{N}} \mathrm{M}(D)$; (ii) $\mathrm{M}\left(\cup_{j \geq 1} \Omega_{j}\right) \geq\left(1-F(c) 2^{-d \mu^{N}}\right) \mathrm{M}(\Omega)$.

Proof. We iterate the map on $\Omega$ for $k(N)$ times. Then there are at most $a^{\left\lfloor\frac{k(N)}{m_{0}}\right\rfloor+1}$ disjoint set $\widehat{\Omega}_{j} \subset \Omega$ such that $T^{k(N)}\left(\widehat{\Omega}_{j}\right)$ is a component of $T^{k(N)}(\Omega)$ for each $j$ (note that the assumption that $\mathrm{M}\left(T^{i}\left(\widehat{\Omega}_{j}\right)\right) \leq \delta_{1} \mathrm{M}(D)$ is valid for each $\widehat{\Omega}_{j}$ and each $\left.i \leq k(N)\right)$. 
Then the total measure of all $\widehat{\Omega}_{j}{ }^{\prime}$ s satisfying $\mathrm{M}\left(T^{k(N)}\left(\widehat{\Omega}_{j}\right)\right) \leq 2^{-\mu^{N}} \mathrm{M}(D)$ is less than

$$
\begin{aligned}
& 2^{-\mu^{N}} \mathrm{M}(D) a^{\left\lfloor\frac{k(N)}{m_{0}}\right\rfloor+1} E_{-}(c)^{-k(N)} \quad\left(\text { since } \mathrm{M}\left(\widehat{\Omega}_{j}\right) \leq E_{-}(c)^{-k(N)} \mathrm{M}\left(T^{k(N)}\left(\widehat{\Omega}_{j}\right)\right)\right) \\
& \leq a 2^{-\mu^{N}} \mathrm{M}(D)\left(\frac{E_{-}(c)}{a^{1 / m_{0}}}\right)^{-k(N)} \\
& \leq a 2^{-\mu^{N}} \mathrm{M}(D)\left(\frac{E_{-}(c)}{a^{1 / m_{0}}}\right)^{\log _{E_{+}(c)} \frac{\mathrm{M}(\Omega)}{\delta_{1} \mathrm{M}(D)} \mid+1} \\
& \leq a 2^{-\mu^{N}} \mathrm{M}(D)\left(\frac{E_{-}(c)}{a^{1 / m_{0}}}\right)^{1-\log _{E_{+}(c)} \delta_{1}}\left(\frac{E_{-}(c)}{a^{1 / m_{0}}}\right)^{-\log _{E_{+}(c)} \mathrm{M}(D)}\left(\frac{E_{-}(c)}{a^{1 / m_{0}}}\right)^{\log _{E_{+}(c)} \mathrm{M}(\Omega)} \\
& =F(c) 2^{-\mu^{N}} \mathrm{M}(D)^{1-\log _{E_{+}(c)}\left(\frac{E_{-}(c)}{a^{1 / m_{0}}}\right)} \mathrm{M}(\Omega)^{\log _{E_{+}(c)}\left(\frac{E_{-}(c)}{a^{1 / m_{0}}}\right)} \quad\left(\text { since } a^{\log _{b} c}=c^{\log _{b} a}\right) \text {. }
\end{aligned}
$$

Hence it possesses a portion of $\Omega$ less than

$$
\begin{aligned}
& F(c) 2^{-\mu^{N}}\left(\frac{\mathrm{M}(\Omega)}{\mathrm{M}(D)}\right)^{\log _{E_{+}(c)}\left(\frac{E_{-}(c)}{a^{1 / m_{0}}}\right)-1} \\
\leq & F(c) 2^{-\mu^{N}}\left(2^{-\mu^{N+1}}\right)^{\log _{E_{+}(c)}\left(\frac{E_{-}(c)}{a^{1 / m}}\right)-1} \leq F(c) 2^{-d \mu^{N}} .
\end{aligned}
$$

Choose $\Omega_{1}, \cdots$, be all $\widehat{\Omega}_{j}$ with a measure larger than $2^{-\mu^{N}} \mathrm{M}(D)$ and the proof is completed.

Corollary 2.1. Let the domain $D, m_{0}, a, \mu, N_{0}$ and $d$ be as in Lemma 2.1. Then there exists a constant $c_{1}>0$ such that for any $\left(\delta_{1}, m_{0}, a\right)$-good curve or measurable set $\Omega \subset D$ with $\mathrm{M}(\Omega) \leq \delta_{1} \mathrm{M}(D)$, there exist disjoint subcurves or measurable subsets $\Omega_{i} \subset \Omega, i=1,2, \ldots$, such that (i) for any $i$, there exists $k(i)$ such that $T^{k(i)}\left(\Omega_{i}\right)$ is a component of $T^{k(i)}(\Omega)$ and $\mathrm{M}\left(T^{k(i)}\left(\Omega_{i}\right)\right) \geq 2^{-\mu^{N_{0}}} \mathrm{M}(D)$; (ii) $\mathrm{M}\left(\cup_{i} \Omega_{i}\right) \geq c_{1} \mathrm{M}(\Omega)$.

Proof. Let $N$ be the unique integer such that

$$
2^{-\mu^{N+1}} \mathrm{M}(D) \leq \mathrm{M}(\Omega)<2^{-\mu^{N}} \mathrm{M}(D)
$$

and denote $\Omega_{N+1}=\Omega$. Applying Iteration Lemma 2.1 on $\Omega_{N+1}$, there exist disjoint $\Omega_{N+1}^{i} \subset \Omega_{N+1}, i=1, \ldots$, such that $(a)_{N+1} T^{k(N)}\left(\Omega_{N+1}^{i}\right)$ is a component of $T^{k(N)}\left(\Omega_{N+1}\right)$ and $\mathrm{M}\left(T^{k(N)}\left(\Omega_{N+1}^{i}\right)\right) \geq 2^{-\mu^{N}} \mathrm{M}(D)$ for each $i ;(b)_{N+1} \mathrm{M}\left(\cup_{i \geq 1} \Omega_{N+1}^{i}\right) \geq$ $\left(1-F(c) 2^{-d \mu^{N}}\right) \mathrm{M}\left(\Omega_{N+1}\right)$.

Let

$$
I_{N}=\left\{i \mid \mathrm{M}\left(T^{k(N)}\left(\Omega_{N+1}^{i}\right)\right) \in\left[2^{-\mu^{N}} \mathrm{M}(D), 2^{-\mu^{N-1}} \mathrm{M}(D)\right)\right\}
$$

and denote the set of all other $i$ by $I_{N}^{\prime}$. Let $i \in I_{N}$ and applying Iteration Lemma 2.1 on $T^{k(N)}\left(\Omega_{N+1}^{i}\right)$ and we have that there exist disjoint subcurves or measurable subsets $\Omega_{N}^{i, j} \subset \Omega_{N+1}^{i}, j=1, \cdots$ and $k_{i}(N)$ such that $(a)_{N}^{i} T^{k_{i}(N)}\left(\Omega_{N}^{i, j}\right)$ is a component 
of $T^{k_{i}(N)}\left(\Omega_{N+1}^{i}\right)$ and $\mathrm{M}\left(T^{k_{i}(N)}\left(\Omega_{N}^{i, j}\right)\right) \geq 2^{-\mu^{N-1}} \mathrm{M}(D)$ for each $j ;(b)_{N}^{i} \mathrm{M}\left(\cup_{j \geq 1} \Omega_{N}^{i, j}\right) \geq$ $\left(1-F(c) 2^{-d \mu^{N-1}}\right) \mathrm{M}\left(\Omega_{N+1}^{i}\right)$. Thus we have

$$
\mathrm{M}\left(\cup_{i \in I_{N}}\left(\cup_{j} \Omega_{N}^{i, j}\right) \cup\left(\cup_{i \in I_{N}^{\prime}} \Omega_{N+1}^{i}\right)\right) \geq\left(1-F(c) 2^{-d \mu^{N}}\right)\left(1-F(c) 2^{-d \mu^{N-1}}\right) \mathrm{M}\left(\Omega_{N+1}\right) .
$$

Moreover, the sets on the left hand side in the above inequality are disjoint with each other, and for each set $\widetilde{\Omega}$ of them there exists a $k(\widetilde{\Omega})$ such that $T^{k(\widetilde{\Omega})}$ is a component and $\mathrm{M}\left(T^{k(\widetilde{\Omega})}(\widetilde{\Omega})\right) \geq 2^{-\mu^{N-1}} \mathrm{M}(D)$.

By induction, we can obtain the existence of $\Omega_{i}, i=1, \cdots$, such that (i) and (ii) hold true, where

$$
c_{1}=\prod_{j=N_{0}}^{N}\left(1-F(c)\left(2^{d}\right)^{-\mu^{j}}\right),
$$

which has a positive lower bound for all $N$. In fact, it is sufficient to prove that

$$
\prod_{j=N_{0}}^{\infty}\left(1-F(c)\left(2^{d}\right)^{-\mu^{j}}\right)>0,
$$

which can be obtained from the fact that

$$
\sum_{j=N_{0}}^{\infty} \ln \left(1-F(c)\left(2^{d}\right)^{-\mu^{j}}\right) \geq-F(c) \sum_{j=N_{0}}^{\infty}\left(2^{d}\right)^{-\mu^{j}}>-\infty
$$

(bounded below). This completes the proof.

Remark 2.1. Corollary 2.1 holds true for any

$$
1<\mu<\left(1-\log _{E_{+}(c)} \frac{E_{-}(c)}{a^{1 / m_{0}}}\right)^{-1} .
$$

As $\mu \rightarrow 1$, we obtain the upper bound $\log _{E_{+}(c)}\left(E_{-}(c) a^{-1 / m_{0}}\right) F(c)^{-1}$ for $2^{-\mu^{N_{0}}}$.

\section{The ordered part}

In this section, we will prove the ordered part of Theorem 1.2, that is, we will prove (1.3a) holds true for almost every initial point. For this purpose, it is sufficient to prove the following theorem.

Theorem 3.1. For any given $\epsilon>0$ and almost every initial point $\left(x_{1}(0), x_{2}(0)\right)$ in $[0,1]^{2}$, it holds that $\inf _{n \in \mathbb{N}} \operatorname{dist}\left(\left(x_{1}(n), x_{2}(n)\right), D_{\text {syn }}\right)<\epsilon$.

Remark 3.1. Theorem 3.1 implies that for almost every point, its orbit will enter the $\epsilon$ neighborhood of the diagonal $x_{1}=x_{2}$ for at least one time. We will use it to prove that the orbit of almost every point will enter into (or stay in) the $\epsilon$-neighborhood of the diagonal $x_{1}=x_{2}$ for infinitely many times, which is just (1.3a). 
Proof of (1.3a) from Theorem 3.1. For any positive integer $i$, let

$$
D_{i}=\left\{\left(x_{1}^{(i)}(0), x_{2}^{(i)}(0)\right) \in[0,1]^{2} \mid \operatorname{dist}\left(\left(x_{1}^{(i)}(n), x_{2}^{(i)}(n)\right), D_{\text {syn }}\right) \geq \frac{1}{i} \text { for all } n \in \mathbb{N}\right\} .
$$

Obviously, to obtain (1.3a), it is sufficient to prove that $\mathrm{M}\left(\cup_{i \geq 1} D_{i}\right)=0$ (note that $D_{\text {syn }}$ is an invariant set). Setting $\epsilon=\frac{1}{i}$ in Theorem 3.1, we obtain that $\mathrm{M}\left(D_{i}\right)=0$, which immediately implies $\mathrm{M}\left(\cup_{i \geq 1} D_{i}\right)=0$.

Let

$$
G_{\epsilon}=\left\{\mathbf{p}=\left(x_{1}, x_{2}\right) \in[0,1]^{2} \mid \operatorname{dist}\left(\mathbf{p}, D_{\text {syn }}\right) \leq \epsilon\right\} \quad \text { and } \quad B_{\epsilon}=[0,1]^{2} \backslash G_{\epsilon} .
$$

Theorem 3.1 is a corollary of the following lemma.

Lemma 3.1. There exists a constant $c_{1}>0$ such that for any $0 \leq c<c_{1}$ and $\epsilon>0$, there exists $c_{0} \equiv c_{0}(c, \epsilon)>0$ such that any segment $\Gamma$ with a slope \pm 1 in $[0,1]^{2}$ has disjoint subsegments $\Gamma_{1}, \Gamma_{2}, \cdots$ satisfying (i) for any $i$, there exists $l_{i}$ such that $T^{l_{i}}\left(\Gamma_{i}\right) \subset G_{\epsilon} ;(i i) \mathrm{M}\left(\cup_{i} \Gamma_{i}\right) \geq c_{0} \mathrm{M}(\Gamma)$.

Proof of Theorem 3.1 from Lemma 3.1. From Lemma 3.1, we have that for any segment $\Gamma$ with a slope \pm 1 in $[0,1]^{2}$, there exist disjoint subsegments $\Gamma_{1}, \Gamma_{2}, \cdots$, satisfying (i) and (ii). Obviously $\Gamma \backslash\left(\cup_{i} \Gamma_{i}\right)$ is composed of a collection of disjoint subsegments of $\Gamma$, which is denoted by $\cup_{j} \Gamma_{j}^{\prime}$. From (ii), we know that $\mathrm{M}\left(\cup_{j} \Gamma_{j}^{\prime}\right) \leq\left(1-c_{0}\right) \mathrm{M}(\Gamma)$.

Applying Lemma 3.1 again on each $\cup_{j} \Gamma_{j}^{\prime}$, we obtain that for each $j$, there exist disjoint subsegments $\Gamma_{j, k}^{\prime} \subset \Gamma_{j}^{\prime}, k=1, \ldots$, satisfying $\mathrm{M}\left(\cup_{k} \Gamma_{j, k}^{\prime}\right) \geq c_{0} \mathrm{M}\left(\Gamma_{j}^{\prime}\right)$ and for any $j, k$, there exists $l(j, k)$ such that $T^{l(j, k)}\left(\Gamma_{j, k}^{\prime}\right) \subset G_{\epsilon}$. It is easy to see that $\cup_{j}\left(\Gamma_{j}^{\prime} \backslash\left(\cup_{k} \Gamma_{j, k}^{\prime}\right)\right)$ is also composed of disjoint subsegments of $\Gamma$ and the total length of them is not larger than $\left(1-c_{0}\right)^{2} \mathrm{M}(\Gamma)$. Inductively, we can obtain that for any $i$, we can find disjoint segments $\Gamma_{i, k} \subset \Gamma, k=1,2, \cdots$, such that for each $k$ there exists $l(i, k)$ such that $T^{l(i, k)}\left(\Gamma_{i, k}\right) \subset G_{\epsilon}$ and $\mathrm{M}\left(\Gamma \backslash \cup_{k} \Gamma_{i, k}\right) \leq\left(1-c_{0}\right)^{i} \mathrm{M}(\Gamma)$. Let $i \rightarrow \infty$, we obtain that the set of points in $\Gamma$ whose orbit is always out of $G_{\epsilon}$ is of measure zero. Then from Fubini's Theorem, we obtain Theorem 3.1.

The proof of Lemma 3.1 can be reduced to the following two propositions.

Proposition 3.1. There exists a constant $c_{1}>0$, such that for any $0 \leq c<c_{1}$, there is a $\hat{c}_{0} \equiv \hat{c}_{0}(c)>0$, for any segment $\Gamma_{0}$ in one of four small squares of $[0,1]^{2}$ with a slope \pm 1 and a length less than $\delta_{1}=2^{-16}$, there exists a collection of disjoint subsegments $\Gamma_{i}, i=1,2, \ldots$, satisfying $\sum_{i} \mathrm{M}\left(\Gamma_{i}\right) \geq \hat{c}_{0} \mathrm{M}\left(\Gamma_{0}\right)$ such that for each segment $\Gamma_{i}$, there exists some $l\left(\Gamma_{i}\right)$ such that $T^{l\left(\Gamma_{i}\right)}\left(\Gamma_{i}\right)$ is a component of $T^{l\left(\Gamma_{i}\right)}\left(\Gamma_{0}\right)$ and $T^{l\left(\Gamma_{i}\right)}\left(\Gamma_{i}\right)$ is a segment with a slope \pm 1 and a length larger than $\delta_{1}$.

Proof. From the condition on $\Gamma_{0}$ and the expansion of $T$, we have that $\mathrm{M}\left(T\left(\Gamma_{0}\right)\right) \geq 2(1-$ 2c) $\mathrm{M}\left(\Gamma_{0}\right)$ From the definition of $T$, we know that for small $c$, number of components for short segments increases very slowly as the iterations go forward. For example, it 
can be easily seen that there are disjoint sets $\widehat{\Gamma}_{i}, i=1,2,3,4$ such that $\cup_{i=1}^{4} \widehat{\Gamma}_{i}=\Gamma_{0}$ and $T^{6}\left(\widehat{\Gamma}_{i}\right), i=1,2,3,4$ are all the components of $T^{6}\left(\Gamma_{0}\right)$ (note that for small $c$, the image of $x_{1}, x_{2}=\frac{1}{2}$ under $T$ are close to $x_{1}=1$ and $x_{2}=1$, respectively. In addition, the images of $x_{1}, x_{2}=1$ under $T^{i}$ for $i \leq 6$ is far from $x_{1}, x_{2}=\frac{1}{2}$ ). Applying Iteration Lemma 2.1 and Corollary 2.1 with $m_{0}=6, a=4, \mu=2, N_{0}=4$ and $D=[0,1]^{2}$, the conclusion is obtained.

Proposition 3.2. There exists a constant $c_{1}>0$ such that for any $0 \leq c<c_{1}$ and $\epsilon>0$, there exists $c_{2} \equiv c_{2}(c, \epsilon)>0$ such that for any segment $\Gamma_{0}$ in some small square of $[0,1]^{2}$ with a slope \pm 1 and a length larger than $\delta_{1}=2^{-16}$, there exists a segment $\Gamma \subset \Gamma_{0}$ and $l$ such that $T^{l}(\Gamma) \subset G_{\epsilon}$ and $\mathrm{M}(\Gamma) \geq c_{2} \mathrm{M}\left(\Gamma_{0}\right)$.

Proof. We first claim that there exists a constant $e>0$ such that for any $\Gamma_{0}$ in some small square with a slope \pm 1 and a length larger than $\delta_{1}=2^{-16}$, there exists a segment $\Gamma_{1}$ with a slope \pm 1 in the curve $T\left(\Gamma_{0}\right)$ or $T^{2}\left(\Gamma_{0}\right)$ such that $\Gamma_{1}$ is in some small squares and $\mathrm{M}\left(\Gamma_{1}\right) \geq(1+e) \mathrm{M}\left(\Gamma_{0}\right)$.

Since $\mathrm{M}\left(T\left(\Gamma_{0}\right)\right) \geq 2(1-2 c) \mathrm{M}\left(\Gamma_{0}\right)$, if $T\left(\Gamma_{0}\right)$ is in some small square, the claim is proved by setting $\Gamma_{1}=T\left(\Gamma_{0}\right)$.

Thus we consider the case that the intersection between $T\left(\Gamma_{0}\right)$ and $x_{2}=\frac{1}{2}$ (or $x_{1}=\frac{1}{2}$ ) is nonempty. If both $x_{1}=\frac{1}{2}$ and $x_{2}=\frac{1}{2}$ have an intersection set with $T\left(\Gamma_{0}\right)$, then it is not difficult to see that $T\left(\Gamma_{0}\right)$ has an intersection set with $x_{1}+x_{2}=1$, this ends the proof of this proposition. Hence without loss of generality we assume $T\left(\Gamma_{0}\right)$ only crosses $x_{2}=\frac{1}{2}$ (or $x_{1}=\frac{1}{2}$ ).

Let $\Gamma_{1,1}$ and $\Gamma_{1,2}$ be the components of $T\left(\Gamma_{0}\right)$ and $e=\frac{\lambda^{2}}{\lambda+1}-1$ with $\lambda=2(1-2 c)$. It is obvious that $e>0$ for small $c$.

From the expansibility of $T$, we have

$$
\mathrm{M}\left(\Gamma_{1,1}\right)+\mathrm{M}\left(\Gamma_{1,2}\right)=\mathrm{M}\left(T\left(\Gamma_{0}\right)\right) \geq \lambda \mathrm{M}\left(\Gamma_{0}\right) .
$$

If

$$
\mathrm{M}\left(\Gamma_{1,1}\right) \geq(1+e) \mathrm{M}\left(\Gamma_{0}\right) \quad \text { or } \quad \mathrm{M}\left(\Gamma_{1,2}\right) \geq(1+e) \mathrm{M}\left(\Gamma_{0}\right),
$$

the claim is proved by choosing $\Gamma_{1}$ to be $\Gamma_{1,1}$ or $\Gamma_{1,2}$.

Thus, in the following, we consider the case that both $\Gamma_{1,1}$ and $\Gamma_{1,2}$ are shorter than $(1+e) \mathrm{M}\left(\Gamma_{0}\right)$. From (3.1) we have both $\Gamma_{1,1}$ and $\Gamma_{1,2}$ are longer than $(\lambda-(1+e)) \mathrm{M}\left(\Gamma_{0}\right)$.

Let $T^{2}\left(\Gamma_{0}\right)=\Gamma_{2,1} \cup \Gamma_{2,2}$ with $\Gamma_{2,1} \cap \Gamma_{2,2}$ being a one-point set, where $\Gamma_{2, i}=T\left(\Gamma_{1, i}\right)$ with a slope $(-1)^{i+1}, i=1,2$ (see Fig. 1). By a direct computation, it holds that

$$
\mathrm{M}\left(\Gamma_{2, i}\right) \geq \lambda \mathrm{M}\left(\Gamma_{1, i}\right) \geq \lambda(\lambda-(1+e)) \mathrm{M}\left(\Gamma_{0}\right)=(1+e) \mathrm{M}\left(\Gamma_{0}\right) .
$$

Then if $\Gamma_{2,1}$ or $\Gamma_{2,2}$ is in some small square, we complete the proof of the claim.

So we assume both $\Gamma_{2,1}$ and $\Gamma_{2,2}$ are not in some small square. In the following, we will prove it is impossible. Recall that the slopes of these two segments are 1 and -1 , respectively (see Fig. 1). Moreover, it is clear that the lines where $\Gamma_{2,1}$ and $\Gamma_{2,2}$ lie in are 


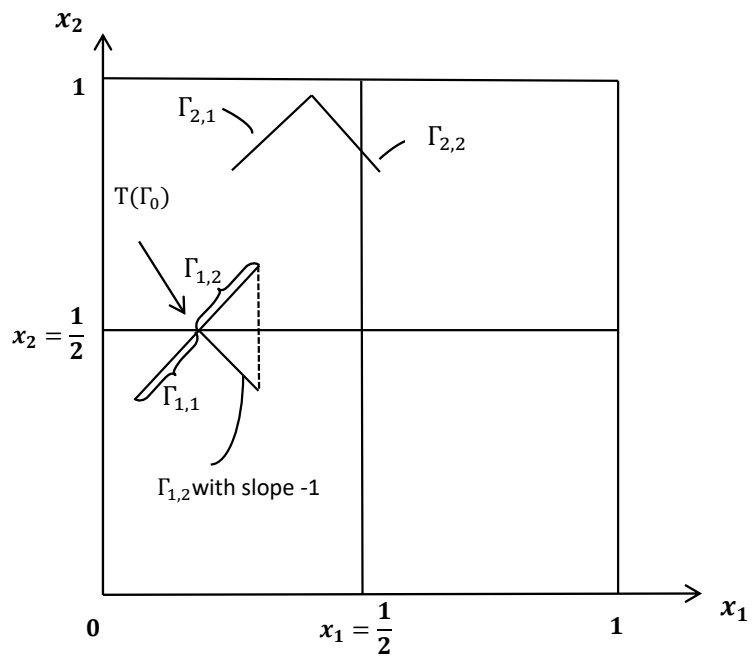

Figure 1: Segments of $\Gamma_{1}=T\left(\Gamma_{0}\right)$ and $\Gamma_{2}=T^{2}\left(\Gamma_{0}\right)=\Gamma_{2,1} \cup \Gamma_{2,2}$.

symmetric with respect to a vertical line, which implies that it is impossible that both $\Gamma_{2,1}$ and $\Gamma_{2,2}$ have an intersection with $x_{1}=\frac{1}{2}$ ( or $x_{2}=\frac{1}{2}$ ). This ends the proof of the claim.

By induction, if $\Gamma_{i}$ has no intersection with $x_{1}=x_{2}$ for $i \geq 0$, we can find a segment $\Gamma_{i+1} \subset T\left(\Gamma_{i}\right)$ or $T^{2}\left(\Gamma_{i}\right)$ lying in some small square satisfies $\mathrm{M}\left(\Gamma_{i+1}\right) \geq(1+e) \mathrm{M}\left(\Gamma_{i}\right)$.

Since the set $[0,1]^{2}$ has a finite diameter, there exists some $i_{0} \leq\left\lfloor-\log _{1+e} \mathrm{M}\left(\Gamma_{0}\right)\right\rfloor+1$ such that $\Gamma_{i_{0}}$ has an intersection with $x_{1}=x_{2}$ satisfying $\mathrm{M}\left(\Gamma_{i_{0}} \cap G_{\epsilon}\right) \geq \epsilon$. Since $\mathrm{M}\left(T^{-1}(\Gamma)\right) \geq 2^{-1} \mathrm{M}(\Gamma)$ for any curve $\Gamma$ in some small square and $\mathrm{M}\left(\Gamma_{0}\right) \leq \frac{\sqrt{2}}{2}$, we have that

$$
\frac{\mathrm{M}\left(T^{-i_{0}}\left(\Gamma_{i_{0}} \cap G_{\epsilon}\right)\right)}{\mathrm{M}\left(\Gamma_{0}\right)} \geq \sqrt{2} 2^{-i_{0}} \epsilon \equiv c_{2} .
$$

This completes the proof of the proposition.

Remark 3.2. With a smaller $\delta_{1}$, the same conclusion holds true for larger $c$, since the frequency for the occurrence of fold (i.e., the segment has nonempty intersection with $x_{1}=\frac{1}{2}$ or $x_{2}=\frac{1}{2}$ ) tends to 0 as $\delta_{1} \rightarrow 0$. In fact, let $\delta_{1} \rightarrow 0$, the upper bound for $c$ tends to $\frac{1}{4}$.

\section{The disordered part}

In this section, we will prove (1.3b), the disordered part of the main theorem, which states that for almost every point in the phase space, its orbit, although will visit any neighborhood of $D_{\text {syn }}$, will also be far away from $D_{\text {syn }}$ for infinitely many times.

It is sufficient to prove that 
Theorem 4.1. For almost every point $\left(x_{1}(0), x_{2}(0)\right)$ in the phase space, there exists an $n=$ $n\left(x_{1}(0), x_{2}(0)\right)$ such that $\left(x_{1}(n), x_{2}(n)\right) \in B_{\gamma_{0}}$ with $\gamma_{0}=2^{-20}$.

Proof of (1.3b) by Theorem 4.1. Let

$$
S_{0}=\left\{\left(x_{1}(0), x_{2}(0)\right) \in[0,1]^{2} \mid\left(x_{1}(i), x_{2}(i)\right) \in G_{\gamma_{0}} \text { for all } i\right\} .
$$

Then from Theorem 4.1, we have that $\mathrm{M}\left(S_{0}\right)=0$. Let

$$
S_{n}=\left\{\left(x_{1}(0), x_{2}(0)\right) \in[0,1]^{2} \mid\left(x_{1}(i), x_{2}(i)\right) \in G_{\gamma_{0}} \text { for all } i>n\right\}
$$

be the subset of $[0,1]^{2}$ such that for each point $\mathbf{p}$ in it and each $i>n, T^{i}(\mathbf{p})$ always stay in $G_{\gamma_{0}}$. From the definition, we have that $T^{n+1}\left(S_{n}\right) \subset S_{0}$. If $\mathrm{M}\left(S_{n}\right)>0$, then there exists $\widetilde{S}_{n} \subset S_{n}$ with $\mathrm{M}\left(\widetilde{S}_{n}\right)>0$ such that $T^{n+1}: \widetilde{S}_{n} \rightarrow T^{n+1}\left(\widetilde{S}_{n}\right) \subset S_{0}$ is a diffeomorphism. It implies

$$
\mathrm{M}\left(S_{0}\right) \geq \mathrm{M}\left(T^{n+1}\left(\widetilde{S}_{n}\right)\right)>0 .
$$

This contradicts the fact that $\mathrm{M}\left(S_{0}\right)=0$. Hence $\mathrm{M}\left(S_{n}\right)=0$ for each $n$, which leads to (1.3b).

From Fubini's Theorem, one can easily see that Theorem 4.1 can be reduced to the following statement: for almost every segment with a slope \pm 1 in the phase space, almost every point on it will be mapped into $B_{\gamma_{0}}$ in a finite time. Thus, it is sufficient to prove the following lemma.

Lemma 4.1. There exists a constant $c_{1}>0$ such that for any $0 \leq c<c_{1}$, there exists $0 \leq c_{3} \equiv$ $c_{3}(c)<1$ such that for almost each segment $\Gamma$ with a slope \pm 1 in $[0,1]^{2}$, there exist its disjoint subsegments $\Gamma_{1}, \Gamma_{2}, \cdots$, satisfying (i) for any $i$, there exists $l_{i} \geq 0$ such that $T^{l_{i}}\left(\Gamma_{i}\right) \subset B_{\gamma_{0}}$; (ii) $\mathrm{M}\left(\cup_{i} \Gamma_{i}\right) \geq c_{3} \mathrm{M}(\Gamma)$.

The proof of Lemma 4.1 can be divided into two propositions.

Proposition 4.1. There exists a constant $c_{1}>0$ such that for any $0 \leq c<c_{1}$, there exist two constants $c_{4}, \delta_{2}>0$ with the following properties: for almost every segment $\Gamma$ with a slope \pm 1 in some small square of $[0,1]^{2}$, there exist its subsegments $\Gamma_{1}, \Gamma_{2}, \cdots$ satisfying (i) for any $i$, there exists $l\left(\Gamma_{i}\right) \geq 0$ such that $T^{l\left(\Gamma_{i}\right)}\left(\Gamma_{i}\right)$ is a component of $T^{l\left(\Gamma_{i}\right)}(\Gamma)$ and $T^{l\left(\Gamma_{i}\right)}\left(\Gamma_{i}\right)$ is a segment with a slope \pm 1 and a length larger than $\delta_{2}$, or $T^{j}\left(\Gamma_{i}\right) \subset B_{\gamma_{0}}$ for some $j \leq l\left(\Gamma_{i}\right)$; (ii) $\mathrm{M}\left(\cup_{i} \Gamma_{i}\right) \geq c_{4} \mathrm{M}(\Gamma)$

Proposition 4.2. Let $c_{1}, c, \delta_{2}$ be defined as above. Assume $\Gamma_{0}$ is a segment with a slope \pm 1 longer than $\delta_{2}$ in some small square of $[0,1]^{2}$. Then there exist $l \geq 0$ and disjoint subsegments $\widehat{\Gamma}_{1}, \widehat{\Gamma}_{2}, \cdots$ of $\Gamma_{0}$ with

$$
\sum_{i} \mathrm{M}\left(\widehat{\Gamma}_{i}\right) \geq \frac{1}{2} \mathrm{M}\left(\Gamma_{0}\right)
$$

such that $T^{l}\left(\widehat{\Gamma}_{i}\right) \subset B_{\gamma_{0}}$ for each $i$. 
Proof of Proposition 4.1. Recall that

$$
G_{\gamma}=\left\{\mathbf{x} \in[0,1]^{2} \mid \operatorname{dist}\left(\mathbf{x}, D_{\text {syn }}\right) \leq \gamma\right\} \text { for } \gamma>0,
$$

where $D_{\text {syn }}=\left\{\mathbf{x} \in[0,1]^{2} \mid x_{1}=x_{2}\right\}$. Let

$$
\widetilde{G}_{\gamma}=\left\{\mathbf{x} \in G_{\gamma} \mid \operatorname{dist}\left(\mathbf{x},\left\{x_{1}+x_{2}=1\right\}\right) \leq \gamma\right\} .
$$

Define $\delta_{2}=2^{-8}$. Obviously, if $T(\mathbf{x})$ has multi-preimages with $\mathbf{x} \in G_{\gamma_{0}}$, then $\mathbf{x} \in \widetilde{G}_{\gamma_{0}}$.

Let $\Omega$ be a segment in $G_{\gamma_{0}} \cap$ \{some small square in $\left.[0,1]^{2}\right\}$ with a length smaller than $\delta_{2}$ such that $T^{l}(\Omega) \nsubseteq D_{\text {syn }}$ for any $l$. We claim that

Claim. For a segment $\Omega \subset G_{\gamma_{0}}$ stated above, $T^{4}(\Omega)$ has at most two components.

Without loss of generality, suppose $T(\Omega)$ has two components. Then we have $\Omega \cap$ $\widetilde{G}_{\gamma_{0}} \neq \varnothing$. Note that $\left(\frac{1}{2}, \frac{1}{2}\right) \in \widetilde{G}_{\gamma_{0}}$ and the diameter of $\widetilde{G}_{\gamma_{0}}$ is less than $2^{-19}$. From the fact that the spectral radius of $T$ is not larger than 2, we obtain that the length of $T(\Omega)$ is less than $2 \mathrm{M}(\Omega) \leq 2 \delta_{2}=2^{-7}$, which implies $T(\Omega)$ is in the $2^{-6}$-neighborhood of $\left(\frac{1}{2}, \frac{1}{2}\right)$.

Moreover, since $T^{i}\left(\frac{1}{2}, \frac{1}{2}\right)=(0,0)(\bmod 1)$ for $i \geq 1$, we have that $T^{i+1}\left(\widetilde{G}_{\gamma_{0}}\right)$ is in $2^{-2}$ neighborhood of $(0,0)$ for $1 \leq i \leq 4$, which is far from $\widetilde{G}_{\gamma_{0}}$. Then we obtain the claim.

Thus, from the claim and similar to the proof of Proposition 3.1 for the ordered part, we obtain the existence of a constant $c_{4}$ and subsegments $\Gamma_{i}$ of $\Gamma$ with a total measure larger than $c_{4} \mathrm{M}(\Gamma)$ such that for each $\Gamma_{i}$, there exists $l_{i} \geq 0$ such that $T^{l_{i}}\left(\Gamma_{i}\right)$ is a segment with slope \pm 1 longer than $\delta_{2}$ or there exists some $j \leq l_{i}$ such that $T^{j}\left(\Gamma_{i}\right) \subseteq B_{\gamma_{0}}$. Since the measure of preimages of $D_{\text {syn }}$ is zero, the conclusion is obtained.

Remark 4.1. Note that there may be a "triple fold" for $T^{i}(\Omega)$, i.e., $T^{i}(\Omega)$ may have intersection points with the lines $x_{1}=\frac{1}{2}, x_{2}=\frac{1}{2}$ and $x_{1}+x_{2}=1$ simultaneously, thus $T^{i}(\Omega)$ consists of 4 segments. In spite of this, the argument above is still valid.

Proof of Proposition 4.2. If the segment $\Gamma_{0}$ is of slope -1 , since $\mathrm{M}\left(\Gamma_{0}\right)>\delta_{2}>2 \gamma_{0}$, the proof is trivial. Thus we assume $\Gamma_{0}$ is of slope 1 . Denote $d_{\text {syn }}\left(\Gamma_{0}\right) \equiv \operatorname{dist}\left(\Gamma_{0}, D_{\text {syn }}\right)$ and let

$$
N\left(\Gamma_{0}\right)=\left\lfloor\log _{2(1-2 c)} \frac{\gamma_{0}}{\mathrm{~d}_{\mathrm{syn}}\left(\Gamma_{0}\right)}\right\rfloor .
$$

We say $\mathbf{x} \in \Gamma_{0}$ is a $i$-regular point if $T^{j}(\mathbf{x}) \notin \widetilde{G}_{2^{j}(1-2 c)^{j} \mathrm{~d}_{\mathrm{syn}}\left(\Gamma_{0}\right)}$ for each $j \leq i$. For simplicity, we assume that

$$
\Gamma_{0} \subset\left\{\mathbf{x} \in[0,1]^{2} \mid x_{2}-x_{1} \leq-\mathrm{d}_{\text {syn }}\left(\Gamma_{0}\right)\right\} .
$$

Denote

$$
\begin{aligned}
& \Gamma_{1, m}=T\left(\Gamma_{0}\right) \cap \widetilde{G}_{2(1-2 c) \mathrm{d}_{\mathrm{syn}}\left(\Gamma_{0}\right),} \\
& \Gamma_{1, l}=T\left(\Gamma_{0}\right) \cap\left\{\mathbf{x} \notin \widetilde{G}_{2(1-2 c) \mathrm{d}_{\mathrm{syn}}\left(\Gamma_{0}\right)} \mid x_{1}, x_{2} \leq \frac{1}{2}\right\}, \\
& \Gamma_{1, r}=T\left(\Gamma_{0}\right) \cap\left\{\mathbf{x} \notin \widetilde{G}_{2(1-2 c) \mathrm{d}_{\mathrm{syn}}\left(\Gamma_{0}\right)} \mid x_{1}, x_{2} \geq \frac{1}{2}\right\} .
\end{aligned}
$$




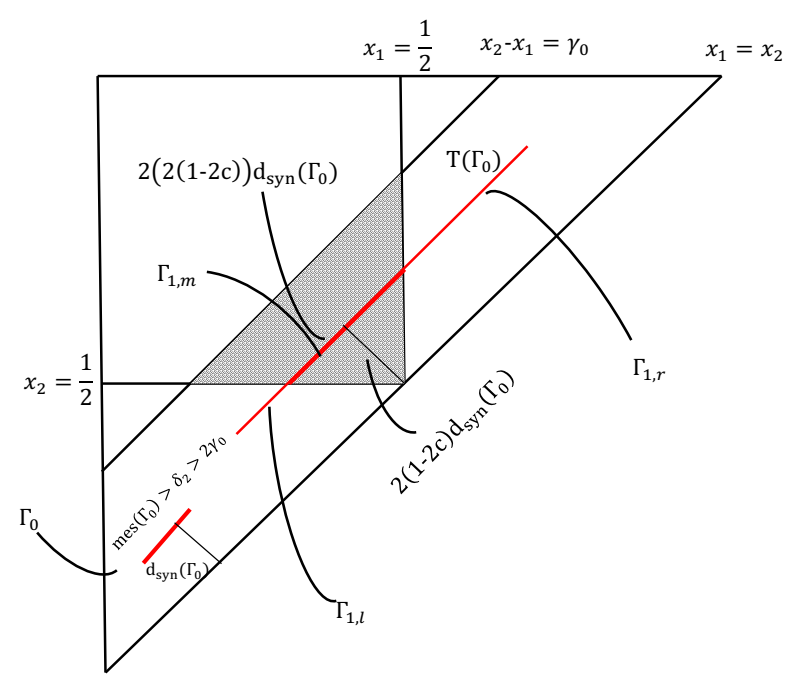

Figure 2: $T\left(\Gamma_{0}\right)=\left(\Gamma_{1, l} \cup \Gamma_{1, r}\right) \cup \Gamma_{1, m} \equiv \Gamma_{1} \cup \Gamma_{1, m}$

Obviously, $\Gamma_{1}=\Gamma_{1, l} \cup \Gamma_{1, r}$ and $\Gamma_{1, m}$, are the image of 1-regular and non-1-regular points in $\Gamma_{0}$ under $T$, respectively. Moreover, $\mathrm{M}\left(T\left(\Gamma_{0}\right)\right) \geq 2 \delta_{2}$. Denote $\mathrm{d}_{\text {syn }}\left(\Gamma_{1}\right) \equiv \operatorname{dist}\left(\Gamma_{1}, D_{\text {syn }}\right)$, which is $2(1-2 c) \mathrm{d}_{\text {syn }}\left(\Gamma_{0}\right)$, since $\Gamma_{1}$ consists of segments with slopes 1 . Since $\Gamma_{1, m} \subset$ $\widetilde{G}_{d_{\text {syn }}\left(\Gamma_{1}\right)}$, we have

$$
\mathrm{M}\left(\Gamma_{1, m}\right) \leq 2(2(1-2 c)) \mathrm{d}_{\mathrm{syn}}\left(\Gamma_{0}\right)
$$

See Fig. 2 for details.

Thus, the ratio $r_{1}$ of 1-regular points in $\Gamma_{0}$ is larger than

$$
\begin{aligned}
& 1-\frac{2(2(1-2 c)) \mathrm{d}_{\text {syn }}\left(\Gamma_{0}\right)}{\mathrm{M}\left(T\left(\Gamma_{0}\right)\right)} \geq \tilde{r}_{1} \equiv 1-\frac{2(1-2 c) \mathrm{d}_{\text {syn }}\left(\Gamma_{0}\right)}{\delta_{2}}, \\
& \mathrm{M}\left(\Gamma_{1}\right) \geq r_{1} \mathrm{M}\left(T\left(\Gamma_{0}\right)\right) \geq 2 \delta_{2} \tilde{r}_{1} .
\end{aligned}
$$

Similarly, denote

$$
\begin{aligned}
& \Gamma_{2, m}=T\left(\Gamma_{1}\right) \cap \widetilde{G}_{2(1-2 c) \mathrm{d}_{\mathrm{syn}}\left(\Gamma_{1}\right)}, \\
& \Gamma_{2, l}=T\left(\Gamma_{1}\right) \cap\left\{\mathbf{x} \notin \widetilde{G}_{2(1-2 c) \mathrm{d}_{\mathrm{syn}}\left(\Gamma_{1}\right)} \mid x_{1}, x_{2} \leq \frac{1}{2}\right\}, \\
& \Gamma_{2, r}=T\left(\Gamma_{1}\right) \cap\left\{\mathbf{x} \notin \widetilde{G}_{2(1-2 c) \mathrm{d}_{\mathrm{syn}}\left(\Gamma_{1}\right)} \mid x_{1}, x_{2} \geq \frac{1}{2}\right\} .
\end{aligned}
$$

Then $\Gamma_{2}=\Gamma_{2, l} \cup \Gamma_{2, r}$ and $\Gamma_{2, m}$, are the images of 1-regular and non-1-regular points in $\Gamma_{1}$ under $T$, respectively. It is clear that the distance

$$
\mathrm{d}_{\text {syn }}\left(\Gamma_{2}\right) \equiv \operatorname{dist}\left(\Gamma_{2}, D_{\text {syn }}\right)
$$


is $(2(1-2 c))^{2} \mathrm{~d}_{\text {syn }}\left(\Gamma_{0}\right)$.

Note that the image of each component of $\Gamma_{1}$ has at most three components. Thus $\Gamma_{2}$ or $\Gamma_{2, m}$ have at most four or two components, respectively. Since $\Gamma_{2, m} \subset \widetilde{G}_{\mathrm{d}_{\mathrm{syn}}\left(\Gamma_{2}\right)}$, it holds that $\mathrm{M}\left(\Gamma_{2, m}\right) \leq 2^{2}(2(1-2 c))^{2} \mathrm{~d}_{\text {syn }}\left(\Gamma_{0}\right)$. Obviously, $\mathrm{M}\left(T\left(\Gamma_{1}\right)\right)=2 \mathrm{M}\left(\Gamma_{1}\right)$.

Thus the ratio of 1-regular points in $\Gamma_{1}$ is larger than $1-\frac{2^{2}(2(1-2 c))^{2} \mathrm{~d}_{\mathrm{syn}}\left(\Gamma_{0}\right)}{\mathrm{M}\left(T\left(\Gamma_{1}\right)\right)}$. It implies that the ratio $r_{2}$ of 2-regular points in $\Gamma_{0}$ is larger than

$$
\begin{aligned}
& r_{1}\left(1-\frac{2^{2}(2(1-2 c))^{2} \mathrm{~d}_{\mathrm{syn}}\left(\Gamma_{0}\right)}{\mathrm{M}\left(T\left(\Gamma_{1}\right)\right)}\right) \geq r_{1}-r_{1} \frac{2^{2}(2(1-2 c))^{2} \mathrm{~d}_{\mathrm{syn}}\left(\Gamma_{0}\right)}{4 \delta_{2} r_{1}} \\
& \quad=r_{1}-\frac{(2(1-2 c))^{2} \mathrm{~d}_{\mathrm{syn}}\left(\Gamma_{0}\right)}{\delta_{2}} \geq 1-\left[2(1-2 c)+(2(1-2 c))^{2}\right] \frac{\mathrm{d}_{\mathrm{syn}}\left(\Gamma_{0}\right)}{\delta_{2}} \equiv \tilde{r}_{2} \\
& \mathrm{M}\left(\Gamma_{2}\right) \geq \mathrm{M}\left(T\left(\Gamma_{1}\right)\right) \tilde{r}_{2} \geq 2^{2} \delta_{2} \tilde{r}_{2} .
\end{aligned}
$$

Inductively, let

$$
\tilde{r}_{i-1}=1-\sum_{j=1}^{i-1}(2(1-2 c))^{j} \frac{\mathrm{d}_{\text {syn }}\left(\Gamma_{0}\right)}{\delta_{2}}
$$

and $\Gamma_{i-1}$ is the segments with slopes 1 of $(i-1)$-regular points in $\Gamma_{0}$ satisfying $\mathrm{M}\left(\Gamma_{i-1}\right) \geq$ $2^{i-1} \delta_{2} \tilde{r}_{i-1}$ and the number of segments in $\Gamma_{i-1}$ is not more than $2^{i-1}$. Moreover, the distance $\mathrm{d}_{\text {syn }}\left(\Gamma_{i-1}\right) \equiv \operatorname{dist}\left(\Gamma_{i-1}, D_{\text {syn }}\right)$ is $(2(1-2 c))^{i-1} \mathrm{~d}_{\text {syn }}\left(\Gamma_{0}\right)$.

Then

$$
\mathrm{M}\left(T\left(\Gamma_{i-1}\right)\right)=2 \mathrm{M}\left(\Gamma_{i-1}\right) \geq 2^{i} \delta_{2} \tilde{r}_{i-1}
$$

and the set of 1-regular and non-1-regular points in $\Gamma_{i-1}$ has at most $2^{i}$ and $2^{i-1}$ components, respectively. Obviously the distance

$$
\mathrm{d}_{\text {syn }}\left(\Gamma_{i}\right) \equiv(2(1-2 c))^{i} \mathrm{~d}_{\text {syn }}\left(\Gamma_{0}\right) .
$$

Let $\Gamma_{i}$ and $\Gamma_{i, m}$ be the image of 1-regular and non-1-regular points in $\Gamma_{i-1}$, respectively. Since $\Gamma_{i, m} \subset \widetilde{G}_{\mathrm{d}_{\mathrm{syn}}\left(\Gamma_{i}\right)}$, we have that the ratio of 1-regular points in $\Gamma_{i-1}$ is larger than $1-\frac{2^{i}(2(1-2 c))^{i} \mathrm{~d}_{\mathrm{syn}}\left(\Gamma_{0}\right)}{2^{i} \delta_{2} \tilde{r}_{i-1}}$. It implies the ratio of $i$-regular points in $\Gamma_{0}$ is larger than

$$
\begin{aligned}
& r_{i-1}\left(1-\frac{(2(1-2 c))^{i} \mathrm{~d}_{\mathrm{syn}}\left(\Gamma_{0}\right)}{\delta_{2} \tilde{r}_{i-1}}\right) \\
\geq & \tilde{r}_{i-1}-\frac{(2(1-2 c))^{i} \mathrm{~d}_{\mathrm{syn}}\left(\Gamma_{0}\right)}{\delta_{2}} \\
= & 1-\sum_{j=1}^{i}(2(1-2 c))^{j} \frac{\mathrm{d}_{\mathrm{syn}}\left(\Gamma_{0}\right)}{\delta_{2}} .
\end{aligned}
$$


Thus, the ratio of $N\left(\Gamma_{0}\right)$-regular points in $\Gamma_{0}$ is larger than

$$
r_{N\left(\Gamma_{0}\right)}=1-\sum_{j=1}^{N\left(\Gamma_{0}\right)}(2(1-2 c))^{j} \frac{\mathrm{d}_{\mathrm{syn}}\left(\Gamma_{0}\right)}{\delta_{2}} .
$$

From the definition of $N\left(\Gamma_{0}\right)$ and the fact that $c$ is small, we have that

$$
\begin{aligned}
1-r_{N\left(\Gamma_{0}\right)} & \leq \frac{2(1-2 c)\left(1-(2(1-2 c))^{N\left(\Gamma_{0}\right)}\right)}{1-2(1-2 c)} \frac{\mathrm{d}_{\mathrm{syn}}\left(\Gamma_{0}\right)}{\delta_{2}} \\
& \leq 4(2(1-2 c))^{N\left(\Gamma_{0}\right)} \frac{\mathrm{d}_{\mathrm{syn}}\left(\Gamma_{0}\right)}{\delta_{2}} \\
& \leq 4 \frac{\gamma_{0}}{\mathrm{~d}_{\mathrm{syn}}\left(\Gamma_{0}\right)} \frac{\mathrm{d}_{\mathrm{syn}}\left(\Gamma_{0}\right)}{\delta_{2}}=\frac{4 \gamma_{0}}{\delta_{2}} \leq \frac{1}{2} \quad \text { if } \gamma_{0}<\frac{\delta_{2}}{8} .
\end{aligned}
$$

Then we know that $r_{N\left(\Gamma_{0}\right)}>\frac{1}{2}$. Moreover, for each $N\left(\Gamma_{0}\right)$-regular point $\mathbf{x}$ in $\Gamma_{0}$, it is not difficult to see that $T^{N\left(\Gamma_{0}\right)+1}(\mathbf{x})$ is in $B_{\gamma_{0}}$, since the distance between $T^{N\left(\Gamma_{0}\right)+1}(\mathbf{x})$ and $D_{\text {syn }}$ is larger than

$$
(2(1-2 c))^{N\left(\Gamma_{0}\right)+1} \mathrm{~d}_{\mathrm{syn}}\left(\Gamma_{0}\right) \geq \gamma_{0}
$$

We complete the proof.

\section{Piecewise expanding case}

In this section, we will prove Theorem 1.3. Recall that the proof of Theorem 1.2 depends heavily on the piecewise-linearity of $T$. In fact, it implies the property that the image of a segment in some small square by $T$ is still a segment, by which the proof can be reduced to the simple fact that a long enough segment in $[0,1]^{2}$ has a nonempty intersection with the line $x_{1}=x_{2}$. Unfortunately, this property is not valid any more with the existence of nonlinear perturbation and we have to deal with curves rather than segments. For a general smooth simple curve $\Gamma$ in $[0,1]^{2}$, no matter how long it is, it may occur that $\Gamma \cap G_{\epsilon}=\varnothing$. To prove Theorem 1.3, we need to exclude the possibility for this troublesome situation. More precisely, we will show that components of a short segment consist of "very flat" simple curves until their length are of constant scale (see case (1iii), (2iii) or (kiii) in the proof of Lemma 5.1). Then everything valid to segments sated above will be also valid to "very flat" simple curves in a similar way.

For this purpose, we need to introduce some quantity to measure how flat a simple curve is. For a point $\mathbf{p}$ in a simple curve $\Gamma$ where the tangent line can be defined, we denote the unit tangent vector of $\Gamma$ at $\mathbf{p}$ by $\mathbf{t}_{\Gamma}(\mathbf{p}) \in \mathbb{R}^{2}$ coinciding with an orientation of the curve. Then we define the range of angles on $\Gamma$ to be

$$
\mathrm{r}_{\mathrm{a}}(\Gamma) \equiv \sup _{\mathbf{p}_{1}, \mathbf{p}_{2} \in \Gamma}\left\|\mathbf{t}_{\Gamma}\left(\mathbf{p}_{2}\right)-\mathbf{t}_{\Gamma}\left(\mathbf{p}_{1}\right)\right\| .
$$


Remark 5.1. Let $[0,1]^{2}=\cup \Gamma_{a}$ is a union of segments, where $\Gamma_{a}$ is a segment with $a$ in some interval $I$. Let $D_{\infty}=\cup_{l=0}^{\infty} T^{-l}\left(D_{\text {syn }}\right)$. Then the measure of $D_{\infty}$ in $[0,1]^{2}$ is zero. Hence from Fubini's theorem, we have that for almost all $a$ in $I, T^{l}\left(\Gamma_{a}\right) \cap D_{\infty}$ is a set of measure zero in $T^{l}\left(\Gamma_{a}\right)$ for any $l$ and in particular,

$$
T^{-l}\left(T^{l}\left(\Gamma_{a}\right) \cap D_{\infty}\right) \cap \Gamma_{a}=\Gamma_{a} \cap T^{-l}\left(D_{\infty}\right)
$$

does not include any open interval. For this reason, each simple curve considered in this section satisfies that the intersection set of it with $D_{\infty}$ is of measure zero.

For any $\mathbf{p} \in[0,1]^{2}$, let $J T(\mathbf{p})$ be the Jacobian matrix of $T$ at $\mathbf{p}$. Then $J T$ is piecewise $C^{1}$ on $\mathbf{p}$. The ordered part in Theorem 1.3 can be reduced to the following three lemmas.

Lemma 5.1. There exist constants $c_{1}, \eta>0$ such that if $0 \leq c<c_{1},\|g\|_{C^{2}} \leq \eta$, there exist constants $c_{5}>0$ and $0<\hat{a}=\mathcal{O}(\eta)$ such that if $\Gamma_{0}$ is a segment in one of small squares in $[0,1]^{2}$ with a slope \pm 1 and a length less than $\delta_{1}$, we can find a collection of sub-curves denoted by $\bar{\Gamma}_{i}$ satisfying ( $i) \sum_{i>0} \mathrm{M}\left(\bar{\Gamma}_{i}\right) \geq c_{5} \mathrm{M}\left(\Gamma_{0}\right)$; (ii) for any $i>0$, there exists $l_{i}$ such that

(a) $T^{l_{i}}\left(\bar{\Gamma}_{i}\right)$ is a component of $T^{l_{i}}\left(\Gamma_{0}\right)$ and $\mathrm{M}\left(T^{l_{i}}\left(\bar{\Gamma}_{i}\right)\right) \geq \delta_{1}$;

(b) $\mathrm{r}_{\mathrm{a}}\left(T^{l_{i}}\left(\bar{\Gamma}_{i}\right)\right) \leq \hat{a} \mathrm{M}\left(T^{l_{i}}\left(\bar{\Gamma}_{i}\right)\right)$.

Proof. Since $T$ is a small perturbation of a (piecewise) linear map satisfying

$$
J T(\mathbf{p})=\left[\begin{array}{cc}
1-c+\eta_{1} & c+\eta_{2} \\
c+\eta_{3} & 1-c+\eta_{4}
\end{array}\right]
$$

with $\left|\eta_{i}\right| \leq \eta$, for $i=1, \cdots, 4$, it is easily seen that $\|J T(\mathbf{p})\| \leq 1+\mathcal{O}(c, \eta)$.

Let $\Gamma_{0}$ be a (short) segment as above. Then $\mathrm{r}_{\mathrm{a}}\left(\Gamma_{0}\right)=0$. With the condition $\|g\|_{C^{2}}$ sufficiently small, it is obvious that if both $x_{1}=\frac{1}{2}$ and $x_{2}=\frac{1}{2}$ have intersections with $T\left(\Gamma_{0}\right)$, then the lemma is immediately proved. Hence, without loss of generality, we assume that $T\left(\Gamma_{0}\right)$ has no intersection with $x_{1}=\frac{1}{2}$.

There are three different cases according to the intersection between $T\left(\Gamma_{0}\right)$ and $x_{2}=\frac{1}{2}$. Case (1i) There is no intersection between $T\left(\Gamma_{0}\right)$ and $x_{2}=\frac{1}{2}$. Denote $\Gamma_{1}=T\left(\Gamma_{0}\right)$. Since $T$ is a small perturbation of a (piecewise) linear map and $\mathrm{M}\left(\Gamma_{0}\right)$ is small, we have that $\mathrm{r}_{\mathrm{a}}\left(\Gamma_{1}\right) \leq a \mathrm{M}\left(\Gamma_{0}\right)$ with $0<a<1$, which is small if $\eta$ is small. In fact, since $\Gamma_{0}$ is a segment, it holds that $\mathbf{t}_{\Gamma_{0}}(\mathbf{p})$ is constant. Then we have

$$
\left\|J T\left(\mathbf{p}_{2}\right)-J T\left(\mathbf{p}_{1}\right)\right\| \leq \max _{p \in \Gamma_{0}}\|D(J T)(p)\|\left\|\mathbf{p}_{2}-\mathbf{p}_{1}\right\| \leq \tilde{a}\left\|\mathbf{p}_{2}-\mathbf{p}_{1}\right\|,
$$

where $D(J T)$ is the Jacobian matrix of $J T$ with respect to $\mathbf{p}$ and $\tilde{a}=\mathcal{O}(\eta) \ll 1$ if $\eta \ll 1$.

Subsequently, because of $\mathbf{t}_{\Gamma_{0}}\left(\mathbf{p}_{1}\right)=\mathbf{t}_{\mathrm{\Gamma}_{0}}\left(\mathbf{p}_{2}\right)$, we obtain that

$$
\begin{gathered}
\left\|\mathbf{t}_{\Gamma_{1}}\left(T\left(\mathbf{p}_{2}\right)\right)-\mathbf{t}_{\Gamma_{1}}\left(T\left(\mathbf{p}_{1}\right)\right)\right\|=\left\|\frac{J T\left(\mathbf{p}_{2}\right) \mathbf{t}_{\Gamma_{0}}\left(\mathbf{p}_{2}\right)}{\left\|J T\left(\mathbf{p}_{2}\right) \mathbf{t}_{\Gamma_{0}}\left(\mathbf{p}_{2}\right)\right\|}-\frac{J T\left(\mathbf{p}_{1}\right) \mathbf{t}_{\Gamma_{0}}\left(\mathbf{p}_{1}\right)}{\left\|J T\left(\mathbf{p}_{1}\right) \mathbf{t}_{\Gamma_{0}}\left(\mathbf{p}_{1}\right)\right\|}\right\| \\
\leq \frac{2}{\min _{p \in \Gamma_{0},\|v\|=1}\|J T(\mathbf{p}) v\|}\left\|J T\left(\mathbf{p}_{2}\right)-J T\left(\mathbf{p}_{1}\right)\right\| \leq a\left\|\mathbf{p}_{2}-\mathbf{p}_{1}\right\|
\end{gathered}
$$




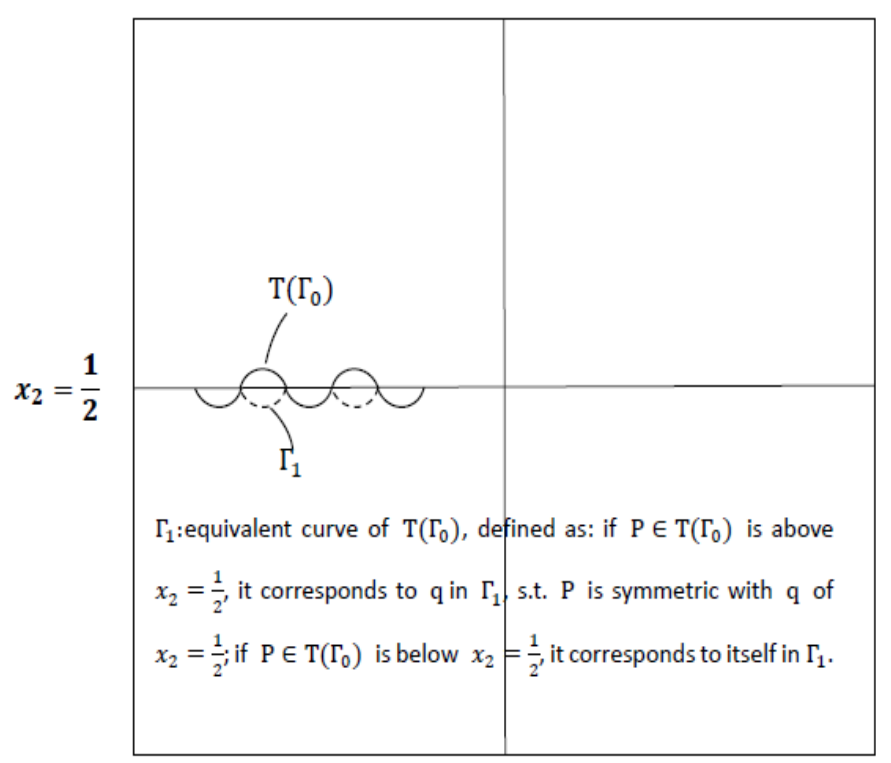

Figure 3: Equivalent curve $\Gamma_{1}$ of $\Gamma_{0}$.

with $a=\mathcal{O}(\eta)$ small.

Case (1ii) There is exactly one intersection point between $T\left(\Gamma_{0}\right)$ and $x_{2}=\frac{1}{2}$. Denote two parts of $T\left(\Gamma_{0}\right)$ divided by the intersection point by $\Gamma_{1,1}$ and $\Gamma_{1,2}$, respectively. Obviously, it holds that $\mathrm{r}_{\mathrm{a}}\left(\Gamma_{1, i}\right) \leq a \mathrm{M}\left(\Gamma_{1, i}\right), i=1,2$ with the same $a$ as in case (1i). Let $\Gamma_{1}$ be any one of these two simple curves, say, $\Gamma_{1,1}$.

Case (1iii) There are more than one intersection points. Same as in case (1i), we have $\mathrm{r}_{\mathrm{a}}\left(T\left(\Gamma_{0}\right)\right) \leq a \mathrm{M}\left(\Gamma_{0}\right)$. Although $T\left(\Gamma_{0}\right)$ has 3 or more components, in the following iterations, it can be replaced by a simple curve in some small square with a small range of angle as follows (note that we have to consider different components separately only if the range of the curve is not small). Define $\Gamma_{1}$ be a piecewise-smooth simple curve in some small square satisfying that $T\left(\Gamma_{1}\right)=T^{2}\left(\Gamma_{0}\right)$ (see Fig. 3). Since there exist at least two intersection points between $T\left(\Gamma_{0}\right)$ and $x_{2}=\frac{1}{2}$, there exist $\mathbf{p}_{1}, \mathbf{p}_{2} \in T\left(\Gamma_{0}\right)$ such that $\mathbf{t}_{T\left(\Gamma_{0}\right)}\left(\mathbf{p}_{1}\right)$ and $\mathbf{t}_{T\left(\Gamma_{0}\right)}\left(\mathbf{p}_{2}\right)$ lies in the upper and lower half planes, respectively. Thus for any point $\mathbf{p}$ in $T\left(\Gamma_{0}\right)$, it holds that

$$
\left\|\mathbf{t}_{T\left(\Gamma_{0}\right)}(\mathbf{p})\right\| \leq \mathrm{r}_{\mathrm{a}}\left(T\left(\Gamma_{0}\right)\right) \leq a \mathrm{M}\left(\Gamma_{0}\right)
$$

Then for any points $\mathbf{p}_{1}, \mathbf{p}_{2}$ in $\Gamma_{1}$, we have

$$
\left\|\mathbf{t}_{\Gamma_{1}}\left(\mathbf{p}_{2}\right)-\mathbf{t}_{\Gamma_{1}}\left(\mathbf{p}_{1}\right) \leq\right\| \mathbf{t}_{\Gamma_{1}}\left(\mathbf{p}_{2}\right)\|+\| \mathbf{t}_{\Gamma_{1}}\left(\mathbf{p}_{1}\right) \| \leq 2 a \mathrm{M}\left(\Gamma_{0}\right) .
$$

Therefore $\mathrm{r}_{\mathrm{a}}\left(\Gamma_{1}\right) \leq 2 a \mathrm{M}\left(\Gamma_{0}\right)$. 
Next we consider three different cases for $T\left(\Gamma_{1}\right)$ according to the intersection between $T\left(\Gamma_{1}\right)$ and $x_{2}=\frac{1}{2}$.

Case (2i) There is no intersection. Denote $\Gamma_{2}=T\left(\Gamma_{1}\right)$. For any nonsingular matrix $A$ and a unit vector $v(\theta)=(\cos \theta, \sin \theta)$ with $\theta \in S^{1}$, we have

$$
\left|\frac{d}{d \theta}(\|A \cdot v(\theta)\|)\right|=\frac{|\operatorname{det}(A)|}{\|A \cdot v(\theta)\|^{2}} .
$$

Thus for $\mathrm{M}\left(\Gamma_{0}\right)$ small, and for any points $\mathbf{p}_{1}, \mathbf{p}_{2}$ in $\Gamma_{1}$, we then have

$$
\begin{aligned}
& \left\|\mathbf{t}_{\Gamma_{2}}\left(T\left(\mathbf{p}_{2}\right)\right)-\mathbf{t}_{\Gamma_{2}}\left(T\left(\mathbf{p}_{1}\right)\right)\right\|=\left\|\frac{J T\left(\mathbf{p}_{2}\right) \mathbf{t}_{\Gamma_{1}}\left(\mathbf{p}_{2}\right)}{\left\|J T\left(\mathbf{p}_{2}\right) \mathbf{t}_{\Gamma_{1}}\left(\mathbf{p}_{2}\right)\right\|}-\frac{J T\left(\mathbf{p}_{1}\right) \mathbf{t}_{\Gamma_{1}}\left(\mathbf{p}_{1}\right)}{\left\|J T\left(\mathbf{p}_{1}\right) \mathbf{t}_{\mathrm{T}_{1}}\left(\mathbf{p}_{1}\right)\right\|}\right\| \\
& \leq\left\|\frac{J T\left(\mathbf{p}_{2}\right) \mathbf{t}_{\Gamma_{1}}\left(\mathbf{p}_{1}\right)}{\left\|J T\left(\mathbf{p}_{2}\right) \mathbf{t}_{\Gamma_{1}}\left(\mathbf{p}_{1}\right)\right\|}-\frac{J T\left(\mathbf{p}_{1}\right) \mathbf{t}_{\Gamma_{1}}\left(\mathbf{p}_{1}\right)}{\left\|J T\left(\mathbf{p}_{1}\right) \mathbf{t}_{\Gamma_{1}}\left(\mathbf{p}_{1}\right)\right\|}\right\|+\left\|\frac{J T\left(\mathbf{p}_{2}\right) \mathbf{t}_{\Gamma_{1}}\left(\mathbf{p}_{2}\right)}{\left\|J T\left(\mathbf{p}_{2}\right) \mathbf{t}_{\mathrm{\Gamma}_{1}}\left(\mathbf{p}_{2}\right)\right\|}-\frac{J T\left(\mathbf{p}_{2}\right) \mathbf{t}_{\mathrm{\Gamma}_{1}}\left(\mathbf{p}_{1}\right)}{\left\|J T\left(\mathbf{p}_{2}\right) \mathbf{t}_{\mathrm{\Gamma}_{1}}\left(\mathbf{p}_{1}\right)\right\|}\right\| \\
& \leq a \mathrm{M}\left(\Gamma_{1}\right)+(1+\mathcal{O}(c, \eta))\left\|\mathbf{t}_{\Gamma_{1}}\left(\mathbf{p}_{2}\right)-\mathbf{t}_{\Gamma_{1}}\left(\mathbf{p}_{1}\right)\right\| \\
& \leq a \mathrm{M}\left(\Gamma_{1}\right)+(1+\mathcal{O}(c, \eta)) \mathrm{r}_{\mathrm{a}}\left(\Gamma_{1}\right) .
\end{aligned}
$$

In the above, we use the fact

$$
\operatorname{det}(J T(P))=4+\mathcal{O}(c, \eta) \text { and }\|J T(P) \cdot v\|=2+\mathcal{O}(c, \eta)
$$

for any $P$ and any unit vector $v$. Therefore, it holds that

$$
\mathrm{r}_{\mathrm{a}}\left(\Gamma_{2}\right) \leq a \mathrm{M}\left(\Gamma_{1}\right)+(1+\mathcal{O}(c, \eta)) \mathrm{r}_{\mathrm{a}}\left(\Gamma_{1}\right) .
$$

Case (2ii) There is exactly one intersection point. Assume $T\left(\Gamma_{1}\right)=\Gamma_{2,1} \cup \Gamma_{2,2}$ and $\Gamma_{2,1} \cap$ $\Gamma_{2,2}$ be the intersection point. For each $\Gamma_{2, i}$, we have a similar estimate for $r_{a}\left(\Gamma_{2, i}\right)$ as in case(2i). We will denote any component of it, say $\Gamma_{2,1}$, by $\Gamma_{2}$.

Case (2iii) There are more than one intersection points. Same as in case (2i), we have

$$
\mathrm{r}_{\mathrm{a}}\left(T\left(\Gamma_{1}\right)\right) \leq a \mathrm{M}\left(\Gamma_{1}\right)+(1+\mathcal{O}(c, \eta)) \mathrm{r}_{\mathrm{a}}\left(\Gamma_{1}\right) .
$$

Since in the simple curve there are at least two points on $x_{2}=\frac{1}{2}$, there exist $\mathbf{p}_{1}, \mathbf{p}_{2} \in T\left(\Gamma_{1}\right)$ such that $\mathbf{t}_{T\left(\Gamma_{1}\right)}\left(\mathbf{p}_{1}\right)$ and $\mathbf{t}_{T\left(\Gamma_{1}\right)}\left(\mathbf{p}_{2}\right)$ lies in the upper and lower half planes, respectively. Thus for any point $\mathbf{p}$ in $T\left(\Gamma_{1}\right)$, it holds that

$$
\left\|\mathbf{t}_{T\left(\Gamma_{1}\right)}(\mathbf{p})\right\| \leq \mathrm{r}_{\mathrm{a}}\left(T\left(\Gamma_{1}\right)\right) \leq a \mathrm{M}\left(\Gamma_{1}\right)+(1+\mathcal{O}(c, \eta)) \mathrm{r}_{\mathrm{a}}\left(\Gamma_{1}\right) .
$$

Let $\Gamma_{2}$ be a piecewise-smooth simple curve in some small square satisfying $T\left(\Gamma_{2}\right)=$ $T^{2}\left(\Gamma_{1}\right)$. Thus for any points $\mathbf{p}_{1}, \mathbf{p}_{2}$ in $\Gamma_{2}$, we have

$$
\begin{aligned}
& \left\|\mathbf{t}_{\Gamma_{2}}\left(\mathbf{p}_{2}\right)-\mathbf{t}_{\Gamma_{2}}\left(\mathbf{p}_{1}\right)\right\| \leq\left\|\mathbf{t}_{\Gamma_{2}}\left(\mathbf{p}_{2}\right)\right\|+\left\|\mathbf{t}_{\Gamma_{2}}\left(\mathbf{p}_{1}\right)\right\| \\
\leq & 2 a \mathrm{M}\left(\Gamma_{1}\right)+2(1+\mathcal{O}(c, \eta)) \mathrm{r}_{\mathrm{a}}\left(\Gamma_{1}\right) .
\end{aligned}
$$


Note that $\lambda=2(1-2 c)$. By induction, we have that for any $k$, in case (ki) and (kii) it holds that

$$
\begin{aligned}
\mathrm{r}_{\mathrm{a}}\left(\Gamma_{k}\right) & \leq a \mathrm{M}\left(\Gamma_{k-1}\right)+(1+\mathcal{O}(c, \eta)) \mathrm{r}_{\mathrm{a}}\left(\Gamma_{k-1}\right) \\
& \leq a \lambda^{-1} \mathrm{M}\left(\Gamma_{k}\right)+(1+\mathcal{O}(c, \eta)) \mathrm{r}_{\mathrm{a}}\left(\Gamma_{k-1}\right) \quad\left(\text { since } \mathrm{M}\left(\Gamma_{k}\right) \geq \lambda \mathrm{M}\left(\Gamma_{k-1}\right)\right),
\end{aligned}
$$

and in case (kiii) we have that

$$
\begin{aligned}
\mathrm{r}_{\mathrm{a}}\left(\Gamma_{k}\right) & \leq 2 a \mathrm{M}\left(\Gamma_{k-1}\right)+2(1+\mathcal{O}(c, \eta)) \mathrm{r}_{\mathrm{a}}\left(\Gamma_{k-1}\right) \\
& \leq 2 a \lambda^{-1} \mathrm{M}\left(\Gamma_{k}\right)+2(1+\mathcal{O}(c, \eta)) \mathrm{r}_{\mathrm{a}}\left(\Gamma_{k-1}\right) .
\end{aligned}
$$

On the other hand, the frequency for the occurrence of case (kiii) is very low. In fact, in case (kiii), (5.2) together with the fact that $\Gamma_{k}$ is short imply that the simple curve $\Gamma_{k}$ "nearly" coincides with the line $x_{1}=\frac{1}{2}$, so it is mapped into a simple curve "nearly" coincides with the line $x_{1}=1$ by $T$. Thus, we may assume that for $j=6 l+6$, (jiii) occurs and thus $\mathrm{r}_{\mathrm{a}}\left(\Gamma_{j}\right)$ should be estimated by (5.2), while for $j=6 l+1, \cdots, 6 l+5$, (jiii) will not occur and $\mathrm{r}_{\mathrm{a}}\left(\Gamma_{j}\right)$ should be estimated by (5.1). Hereafter, we let $g \equiv 1+\mathcal{O}(c, \eta)$. Since $\mathrm{M}\left(\Gamma_{6 l+i}\right) \leq \lambda^{-(6-i)} \mathrm{M}\left(\Gamma_{6 l+6}\right)$ and $\mathrm{r}_{\mathrm{a}}\left(\Gamma_{0}\right)=0$, we have

$$
\begin{aligned}
& \mathrm{r}_{\mathrm{a}}\left(\Gamma_{6(l+1)}\right) \leq 2 a \lambda^{-1} \mathrm{M}\left(\Gamma_{6(l+1)}\right)+2 g r_{a}\left(\Gamma_{6 l+5}\right) \leq \cdots \\
\leq & 2 a \lambda^{-1} \mathrm{M}\left(\Gamma_{6(l+1)}\right)+2 g a \lambda^{-1} \mathrm{M}\left(\Gamma_{6 l+5}\right)+2 g^{2} a \lambda^{-1} \mathrm{M}\left(\Gamma_{6 l+4}\right)+2 g^{3} a \lambda^{-1} \mathrm{M}\left(\Gamma_{6 l+3}\right) \\
& +2 g^{4} a \lambda^{-1} \mathrm{M}\left(\Gamma_{6 l+2}\right)+2 g^{5} r_{a}\left(\Gamma_{6 l}\right) \leq \cdots \\
\leq & 2 a \lambda^{-1}\left(1+\frac{g}{\lambda}+\cdots+\left(\frac{g}{\lambda}\right)^{5}\right)\left(1+2\left(\frac{g}{\lambda}\right)^{6}+\cdots+\left(2\left(\frac{g}{\lambda}\right)^{6}\right)^{l}\right) \mathrm{M}\left(\Gamma_{6(l+1)}\right)+\left(2 g^{6}\right)^{l} \mathrm{r}_{\mathrm{a}}\left(\Gamma_{0}\right) \\
= & 2 a \lambda^{-1}\left(\sum_{i=0}^{5}\left(\frac{g}{\lambda}\right)^{i}\right) \sum_{i=0}^{l}\left(2\left(\frac{g}{\lambda}\right)^{6}\right)^{i} \mathrm{M}\left(\Gamma_{6(l+1)}\right) .
\end{aligned}
$$

For $k=6 l+j, j=1, \cdots, 5$, let

$$
b_{i}=\left(2\left(\frac{g}{\lambda}\right)^{6}\right)^{i}\left(\frac{g}{\lambda}\right)^{j}
$$

Then from (5.3), if $2(g / \lambda)^{6}<1$, we have

$$
\mathrm{r}_{\mathrm{a}}\left(\Gamma_{k}\right) \leq \hat{a} \mathrm{M}\left(\Gamma_{k}\right),
$$

where

$$
\hat{a}=2 a \lambda^{-1} \sum_{i=0}^{5}\left(\frac{g}{\lambda}\right)^{i} \beta
$$

with

$$
\beta=\sum_{i=0}^{\infty} b_{i}<\infty .
$$

With these estimates, we conclude that estimates for current situation is totally similar to the one in the proof for the tent map. In particular, Corollary 2.1 is available and thus we can obtain the lemma. 
In the following we will give the proof for the case $f_{0}(x)=1-s\left|x-\frac{1}{2}\right|, s=2-s_{0}>0$ with $s_{0}>0$. For the case $s_{0}=0$, the proof can be obtained in a similar (in fact simpler) way.

The following lemma can make the argument simpler.

Lemma 5.2. Assume $0<c, \eta \ll s_{0} \ll 1$. For almost every point $\mathbf{p}$ in $[0,1]^{2}$, there exists $a$ $i(\mathbf{p}) \in \mathbb{N}$ such that for all $i \geq i(\mathbf{p}), x_{1}\left(T^{i}(\mathbf{p})\right), x_{2}\left(T^{i}(\mathbf{p})\right) \in\left[\tau_{2}, 1-\tau_{1}\right]$, where

$$
\tau_{1}=\frac{s_{0}}{2}-\eta>0 \quad \text { and } \quad \tau_{2}=(1-c)(s-\eta)\left(\frac{s_{0}}{2}-\eta\right)>0
$$

Proof. If $0<x_{1}(\mathbf{p})<\frac{1}{4}$, then

$$
x_{1}(T(\mathbf{p}))=(1-c) f\left(x_{1}(\mathbf{p})\right)+c f\left(x_{2}(\mathbf{p})\right) \geq(s-\eta)(1-c) x_{1}(\mathbf{p}) .
$$

Thus $\left\{x_{1}\left(T^{i}(\mathbf{p})\right)\right\}_{i}$ is an increasing sequence if only $x_{1}\left(T^{i}(\mathbf{p})\right) \leq \frac{1}{4}$. If $x_{1}(\mathbf{p})>\frac{3}{4}$, then

$$
x_{1}(T(\mathbf{p})) \leq \max _{x \in\left[\frac{3}{4}, 1\right]} f(x)<\frac{3}{4} .
$$

Thus we only need to consider the situation $\frac{1}{4} \leq x_{1}(\mathbf{p}) \leq \frac{3}{4}$. For this case, it holds that

$$
\begin{aligned}
x_{1}(T(\mathbf{p})) & \left.=(1-c) f\left(x_{1}(\mathbf{p})\right)+c f\left(x_{2}(\mathbf{p})\right)\right) \leq \max _{x \in[0,1]} f(x) \\
& \leq \frac{s}{2}+\eta=1-\frac{s_{0}}{2}+\eta=1-\tau_{1} .
\end{aligned}
$$

Subsequently, we have

$$
x_{1}\left(T^{2}(\mathbf{p})\right) \geq(1-c) f\left(\tau_{1}\right) \geq(1-c)(s-\eta)\left(\frac{s_{0}}{2}-\eta\right)=\tau_{2} .
$$

Thus eventually the orbit of $\mathbf{p}$ under the map $T$ lies between $x_{1}=\tau_{2}$ and $x_{1}=1-\tau_{1}$.

Similarly we can obtain the estimate for $x_{2}(\mathbf{p})$. This completes the proof.

From Lemma 5.2, without loss of generality we can replace the phase space $[0,1]^{2}$ by $\left[\tau_{2}, 1-\tau_{1}\right]^{2}$, that is,

$$
x_{1}, x_{2} \in\left[\tau_{2}, 1-\tau_{1}\right] .
$$

Lemma 5.3. There exists $c_{1}>0$ such that for any $0 \leq c<c_{1}$, there exists a fixed number $c_{2}>0$ such that for any piecewise $\mathcal{C}^{2}$-smooth simple curve $\Gamma_{0}$ with a length $\mathrm{M}\left(\Gamma_{0}\right) \in\left[\delta_{1}, 2 \delta_{1}\right]$ and satisfying $\mathrm{r}_{\mathrm{a}}\left(\Gamma_{0}\right) \leq \mathcal{O}(\eta) \mathrm{M}\left(\Gamma_{0}\right)$, there exists a simple curve $\Gamma \subset \Gamma_{0}$ with $\mathrm{M}(\Gamma) \geq c_{2} \mathrm{M}\left(\Gamma_{0}\right)$ and $l(\Gamma) \in \mathbb{N}$ satisfying $T^{l(\Gamma)}(\Gamma) \subset G_{\epsilon}$.

Proof. It can be reduced to the following claim.

Claim. Let $\delta_{1}=2^{-16}$ defined as before. Then there exist a constant $\hat{e}>1$ and $i_{0}$ independent of $\eta$, such that for $\eta \ll 1$, the following holds true: 
1. Assume for each $0 \leq i \leq j \leq N_{0}$ with $N_{0}=\left\lfloor\log _{\hat{e}} 2 \delta_{1}^{-1}\right\rfloor+1$, we have inductively defined piecewise $\mathcal{C}^{2}$-smooth simple curves $\Gamma_{i}$ in some small square with $\Gamma_{i} \subset T^{l(i)}\left(\Gamma_{i-1}\right)$ for some $l(i) \leq i_{0}+3(i \geq 1)$, such that $\mathrm{M}\left(\Gamma_{i}\right) \geq \hat{e} \mathrm{M}\left(\Gamma_{i-1}\right)$ and $\mathrm{r}_{\mathrm{a}}\left(\Gamma_{i}\right) \leq \mathcal{O}(\eta) \mathrm{M}\left(\Gamma_{i}\right)$.

2. If for each $0 \leq i \leq j, \mathrm{M}\left(\Gamma_{i}\right) \leq 2$ and $\Gamma_{i} \cap\left\{x_{1}=x_{2}\right\} \neq \varnothing$, we have that there exist $l(j+1) \leq i_{0}+3$ and a simple curve $\Gamma_{j+1} \subset T^{l(j+1)}\left(\Gamma_{j}\right)$ in some small square such that

(a) $\mathrm{M}\left(\Gamma_{j+1}\right) \geq \hat{e} \mathrm{M}\left(\Gamma_{j}\right)$;

(b) $\mathrm{r}_{\mathrm{a}}\left(\Gamma_{j+1}\right) \leq \mathcal{O}(\eta) \mathrm{M}\left(\Gamma_{j+1}\right)$.

Claim $\Rightarrow$ Lemma 5.3 .

First we prove the existence of a $j_{0} \leq N_{0}$ satisfying $\Gamma_{j_{0}} \cap\left\{x_{1}=x_{2}\right\} \neq \varnothing$. Otherwise, from the claim we have that either there exists $k_{0} \leq N_{0}$ such that $\Gamma_{i}$ is defined for each $0 \leq i \leq k_{0}$ satisfying $\mathrm{M}\left(\Gamma_{k_{0}}\right) \geq 2$ and $\mathrm{M}\left(\Gamma_{i}\right)<2$ for $0 \leq i \leq k_{0}-1$, or for each $0 \leq i \leq N_{0}$, $\Gamma_{i}$ is defined with $\mathrm{M}\left(\Gamma_{i}\right)<2$.

Note that for any simple curve $\Gamma$ in some small square, it holds that

$$
(\lambda-\mathcal{O}(\eta)) \mathrm{M}(\Gamma) \leq \mathrm{M}(T(\Gamma)) \leq(2+\mathcal{O}(\eta)) \mathrm{M}(\Gamma) .
$$

Then for the former case, from (b) in the claim, we have

$$
\begin{aligned}
\mathrm{r}_{\mathrm{a}}\left(\Gamma_{k_{0}}\right) & \leq \mathcal{O}(\eta) \mathrm{M}\left(\Gamma_{k_{0}}\right) \leq \mathcal{O}(\eta) \mathrm{M}\left(T^{l\left(k_{0}\right)}\left(\Gamma_{k_{0}-1}\right)\right) \\
& \leq(2+\mathcal{O}(\eta))^{l\left(k_{0}\right)} \mathcal{O}(\eta) \mathrm{M}\left(\Gamma_{k_{0}-1}\right) \leq 2 \mathcal{O}(\eta)(2+\mathcal{O}(\eta))^{3+i_{0}} .
\end{aligned}
$$

Hence if $\mathcal{O}(\eta) \leq 200^{-1}$, we obtain that $\mathrm{r}_{\mathrm{a}}\left(\Gamma_{k_{0}}\right) \leq 10^{-1}$. But $\mathrm{M}\left(\Gamma_{k_{0}}\right) \geq 2$ and it is clear that there is no such a simple curve in $[0,1]^{2}$. For the latter case, from (a) in the claim, we know that

$$
\mathrm{M}\left(\Gamma_{N_{0}}\right) \geq \hat{e}^{N_{0}} \mathrm{M}\left(\Gamma_{0}\right) \geq \hat{e}^{\left\lfloor\log _{\hat{e}} 2 \delta_{1}^{-1}\right\rfloor+1} \delta_{1} \geq 2 .
$$

This contradicts the assumption that $\mathrm{M}\left(\Gamma_{N_{0}}\right)<2$ and hence we obtain the existence of $j_{0}$. From the definition of $\Gamma_{i}, i \leq j_{0}$, there exists some $n\left(j_{0}\right) \leq\left(i_{0}+3\right) j_{0}$ such that $\Gamma_{j_{0}} \subset T^{n\left(j_{0}\right)}\left(\Gamma_{0}\right)$.

Let $\widetilde{\Gamma}=\Gamma_{j_{0}} \cap G_{\epsilon}$ and define $\Gamma \subset \Gamma_{0}$ such that $T^{n\left(j_{0}\right)}(\Gamma)=\widetilde{\Gamma}$. First we consider the case that $\Gamma_{j_{0}} \subset G_{\epsilon}$, that is, $\widetilde{\Gamma}=\Gamma_{j_{0}}$.

From (a) in the claim, we have $\mathrm{M}\left(\Gamma_{j_{0}}\right) \geq \hat{e}^{j 0} \mathrm{M}\left(\Gamma_{0}\right)$. On the other hand, it holds that

$$
\mathbf{M}(\Gamma) \geq(2+\mathcal{O}(\eta))^{-n\left(j_{0}\right)} \mathbf{M}\left(\Gamma_{j_{0}}\right)
$$

Consequently, we obtain

$$
\mathrm{M}(\Gamma) \geq(2+\mathcal{O}(\eta))^{-\left(i_{0}+3\right) j_{0}} \hat{e}^{j_{0}} \mathrm{M}\left(\Gamma_{0}\right) \geq(2+\mathcal{O}(\eta))^{-\left(i_{0}+3\right) N_{0}} \mathrm{M}\left(\Gamma_{0}\right) .
$$


This leads to the conclusion if

$$
c_{2} \leq(2+\mathcal{O}(\eta))^{-\left(3+i_{0}\right) N_{0}} .
$$

Next we consider the case that $\Gamma_{j_{0}} \nsubseteq G_{\epsilon}$. Obviously $\mathrm{M}(\widetilde{\Gamma}) \geq \epsilon$. Then we have that

$$
\begin{aligned}
\mathrm{M}(\Gamma) & \geq(2+\mathcal{O}(\eta))^{-n\left(j_{0}\right)} \mathrm{M}(\widetilde{\Gamma}) \\
& \geq(2+\mathcal{O}(\eta))^{-\left(3+i_{0}\right) N_{0}} \epsilon \\
& \geq(2+\mathcal{O}(\eta))^{-\left(3+i_{0}\right) N_{0}} \epsilon\left(2 \delta_{1}\right)^{-1} \mathrm{M}\left(\Gamma_{0}\right) .
\end{aligned}
$$

If

$$
c_{2} \leq(2+\mathcal{O}(\eta))^{-\left(3+i_{0}\right) N_{0}} \epsilon\left(2 \delta_{1}\right)^{-1},
$$

we obtain the conclusion. Thus by setting

$$
c_{2}=\min \left\{1, \epsilon\left(2 \delta_{1}\right)^{-1}\right\}(2+\mathcal{O}(\eta))^{-\left(3+i_{0}\right) N_{0}},
$$

we finish the proof.

\section{Proof of the claim}

We consider the following cases.

1. First, we note if both $T\left(\Gamma_{j}\right) \cap\left\{x_{1}=\frac{1}{2}\right\}$ and $T\left(\Gamma_{j}\right) \cap\left\{x_{2}=\frac{1}{2}\right\}$ are nonempty, then $T\left(\Gamma_{j}\right) \cap\left\{x_{1}=x_{2}\right\} \neq \varnothing$ or $T\left(\Gamma_{j}\right) \cap\left\{x_{1}+x_{2}=1\right\} \neq \varnothing$ and hence $T^{2}\left(\Gamma_{j}\right)$ is nonempty, which implies Lemma 5.3 from the argument above. Hence in the following, we will omit the proof for this trivial case and other similar ones (e.g., both $T^{2}\left(\Gamma_{j}\right) \cap\left\{x_{1}=\frac{1}{2}\right\}$ and $T^{2}\left(\Gamma_{j}\right) \cap\left\{x_{2}=\frac{1}{2}\right\}$ are nonempty). In addition, the estimate on the angle is same as the one in Lemma 5.1, we omit the argument on (b) and only focus on the proof of (a).

For nontrivial cases, the method to define $\Gamma_{j+1}$ is a combination of those in Proposition 3.2 and Lemma 5.1. In fact, the difference between here and Proposition 3.2 lies in that $T\left(\Gamma_{j}\right)$ is no longer a segment here and thus $T\left(\Gamma_{j}\right) \cap\left\{x_{1}=\frac{1}{2}\right\}$ (or $T\left(\Gamma_{j}\right) \cap\left\{x_{2}=\frac{1}{2}\right\}$ ) may has two or more points. In addition, the 'average slope' of the simple curve can be arbitrary. In contrast, for the situation in Proposition 3.2, the slope of the segment is \pm 1 and hence the argument there is much simpler.

2. $T\left(\Gamma_{j}\right) \cap\left(\left\{x_{1}=\frac{1}{2}\right\} \cup\left\{x_{2}=\frac{1}{2}\right\}\right)=\varnothing$. Define $\Gamma_{j+1}=T\left(\Gamma_{j}\right)$. Then

$$
\mathrm{M}\left(\Gamma_{j+1}\right) \geq(\lambda-\mathcal{O}(\eta)) \mathrm{M}\left(\Gamma_{j}\right) \quad \text { with } \lambda-\mathcal{O}(\eta)>1 \quad \text { if }|\lambda-2|,|\eta| \ll 1 .
$$

3. $T\left(\Gamma_{j}\right) \cap\left(\left\{x_{1}=\frac{1}{2}\right\}\right.$ has two or more points and $T\left(\Gamma_{j}\right) \cap\left(\left\{x_{2}=\frac{1}{2}\right\}=\varnothing\right.$ (or vice versa). We replace $T\left(\Gamma_{j}\right)$ by a simple (still piecewise $\mathrm{e}^{2}$-smooth) curve $\widehat{T\left(\Gamma_{j}\right)}$ totally in some small square (by reflecting $T\left(\Gamma_{j}\right)$ with respect to $x_{1}=\frac{1}{2}$ and $x_{2}=\frac{1}{2}$, respectively) such that $T\left(T\left(\Gamma_{j}\right)\right)=T\left(\widehat{T\left(\Gamma_{j}\right)}\right)$. Then we define $\Gamma_{j+1}=\widehat{T\left(\Gamma_{j}\right)}$ (although $\Gamma_{j+1}$ is not in the image of $\Gamma_{j}$, for our purpose, $\widehat{T\left(\Gamma_{j}\right)}$ and $T\left(\Gamma_{j}\right)$ are equivalent) and the case is similar to that in Case 2. 
4. $\left.T\left(\Gamma_{j}\right) \cap\left\{x_{2}=\frac{1}{2}\right\}\right)$ is one-point set and $T\left(\Gamma_{j}\right) \cap\left\{x_{1}=\frac{1}{2}\right\}=\varnothing$ (or vise versa). Let $\Gamma_{j+1,1} \cup \Gamma_{j+1,2}$ be two components of $T\left(\Gamma_{j}\right)$, i.e., $\Gamma_{j+1, l}$ is in some small square $(l=1,2)$. It can be divided into the following subclasses.

(a) Assume

$$
\max \left\{\mathrm{M}\left(\Gamma_{j+1,1}\right), \mathrm{M}\left(\Gamma_{j+1,2}\right)\right\} \geq(1+e-\mathcal{O}(\eta)) \mathrm{M}\left(\Gamma_{j}\right), \quad e=\frac{\lambda^{2}}{\lambda+1}-1 .
$$

Without loss of generality, let $\mathrm{M}\left(\Gamma_{j+1,1}\right) \geq \mathrm{M}\left(\Gamma_{j+1,2}\right)$. Then the proof of claim (a) is completed by setting $\Gamma_{j+1}=\Gamma_{j+1,1}$ and $\hat{e}=1+e-\mathcal{O}(\eta)$.

(b) Assume

$$
\max \left\{\mathrm{M}\left(\Gamma_{j+1,1}\right), \mathrm{M}\left(\Gamma_{j+1,2}\right)\right\} \leq(1+e-\mathcal{O}(\eta)) \mathrm{M}\left(\Gamma_{j}\right) .
$$

By (3.1), we have $\mathrm{M}\left(\Gamma_{j+1, l}\right) \geq(\lambda-(1+e)-\mathcal{O}(\eta)) \mathrm{M}\left(\Gamma_{j}\right), l=1,2$. Denote $\widehat{\Gamma}_{j+1, l}=$ $T\left(\Gamma_{j+1, l}\right), l=1,2$. We need to consider the following sub-cases:

i. $\widehat{\Gamma}_{j+1,1} \cap\left(\left\{x_{1}=\frac{1}{2}\right\} \cup\left\{x_{2}=\frac{1}{2}\right\}\right)=\varnothing\left(\right.$ or $\left.\widehat{\Gamma}_{j+1,2} \cap\left(\left\{x_{1}=\frac{1}{2}\right\} \cup\left\{x_{2}=\frac{1}{2}\right\}\right)=\varnothing\right)$. Then it holds that

$$
\begin{aligned}
\mathrm{M}\left(\widehat{\Gamma}_{j+1,1}\right) & \geq(\lambda-\mathcal{O}(\eta)) \mathrm{M}\left(\Gamma_{j+1,1}\right) \\
& \geq(\lambda-\mathcal{O}(\eta))(\lambda-(1+e)-\mathcal{O}(\eta)) \mathrm{M}\left(\Gamma_{j}\right) \\
& \geq(1+e-\mathcal{O}(\eta)) \mathrm{M}\left(\Gamma_{j}\right) \geq \hat{e} \mathrm{M}\left(\Gamma_{j}\right) .
\end{aligned}
$$

Thus it is sufficient to choose $\Gamma_{j+1}=\widehat{\Gamma}_{j+1,1}$.

ii. Both $\widehat{\Gamma}_{j+1,1} \cap\left(\left\{x_{1}=\frac{1}{2}\right\} \cup\left\{x_{2}=\frac{1}{2}\right\}\right)$ and $\widehat{\Gamma}_{j+1,2} \cap\left(\left\{x_{1}=\frac{1}{2}\right\} \cup\left\{x_{2}=\frac{1}{2}\right\}\right)$ are onepoint sets. Note that angles between $\Gamma_{j+1,1}, \Gamma_{j+1,2}$ and a vertical line are nearly equal to each other at the point $\Gamma_{j+1,1} \cap \Gamma_{j+1,2}$, since $\mathrm{r}_{\mathrm{a}}\left(\Gamma_{j}\right) \ll 1$ if $|\eta| \ll 1$ and $\left.T\right|_{\Gamma_{j}}$ is close to a linear map. Subsequently, since $\mathrm{r}_{\mathrm{a}}\left(\Gamma_{j+1,1}\right), \mathrm{r}_{\mathrm{a}}\left(\Gamma_{j+1,2}\right) \ll 1$ if $|\eta| \ll 1$, we have that angles between the tangent line at any point of $\Gamma_{j+1,1}$ or $\Gamma_{j+1,2}$ and a vertical line are nearly two constants which are nearly equal to each other. In other words, $\Gamma_{j+1,1}$ and $\Gamma_{j+1,2}$ almost lie in two lines symmetric corresponding to a vertical line. The case for $\widehat{\Gamma}_{j+1,1}$ and $\widehat{\Gamma}_{j+1,2}$ is similar if $|\eta| \ll 1$. Without loss of generality, it is sufficient to consider the following two subcases.

A. Both $T\left(\Gamma_{j+1, l}\right) \cap\left\{x_{1}=\frac{1}{2}\right\}, l=1,2$ are nontrivial one-point set. For this case, we have $\widehat{\Gamma}_{j+1, l}(l=1,2)$ almost lie in two lines which are very close to the vertical line $x_{1}=\frac{1}{2}$. It implies that $T\left(\widehat{\Gamma}_{j+1, l}\right)$ almost coincides with the boundary $x_{1}=1-\tau_{1}$.

If $T\left(\widehat{\Gamma}_{j+1,1}\right) \cap\left\{x_{2}=\frac{1}{2}\right\}=\varnothing$, then the argument is completed by setting $\Gamma_{j+1}=$ $T\left(\widehat{\Gamma}_{j+1,1}\right)$.

Consider the case $T\left(\widehat{\Gamma}_{j+1,1}\right) \cap\left\{x_{2}=\frac{1}{2}\right\} \neq \varnothing$. Denote components of $T\left(\widehat{\Gamma}_{j+1,1}\right)$ by $\widetilde{\Gamma}_{j+1, l}, l=1,2$. Obviously, $T\left(\widetilde{\Gamma}_{j+1, l}\right)$ is close to $x_{1}=\tau_{2} \ll 1$ and thus has no 
intersection with $x_{1}=\frac{1}{2}$. Hence we only need to consider the intersection of it with $x_{2}=\frac{1}{2}$. It thus can be reduced to the following subcase.

B. $T\left(\Gamma_{j}\right) \cap\left\{x_{2}=\frac{1}{2}\right\}$ and $\left(\widehat{\Gamma}_{j+1,1}=T\left(\Gamma_{j+1,1}\right)\right) \cap\left\{x_{2}=\frac{1}{2}\right\}$ are nontrivial one-point set, which are denoted by $\mathbf{p}_{j}$ and $\mathbf{p}_{j+1}$, respectively. Obviously, $\mathbf{p}_{j} \in \Gamma_{j+1,1}$. From the fact that $T$ is a perturbation of uncoupled tent map (with a slope $s=2-s_{0}$ satisfying $1 \gg s_{0} \gg \eta \geq 0$ ), we have that

$$
\left|x_{2}\left(T\left(\mathbf{p}_{j}\right)\right)-1\right|=\mathcal{O}\left(\max \left\{s_{0}, c_{1}, \eta\right\}\right) \equiv \mathcal{O}\left(\tau_{0}\right) .
$$

Obviously $x_{2}\left(\mathbf{p}_{j+1}\right)=\frac{1}{2}$, hence $x_{2}\left(T\left(\mathbf{p}_{j+1}\right)\right)=1-\mathcal{O}\left(\tau_{0}\right)$. Similarly, $x_{2}\left(T^{2}\left(\mathbf{p}_{j}\right)\right)=$ $\mathcal{O}\left(\tau_{0}\right)$. Since $\left.T^{2}\left(\mathbf{p}_{j}\right), T\left(\mathbf{p}_{j+1}\right) \in T\left(\widehat{\Gamma}_{j+1,1}\right)\right)=\widetilde{\Gamma}_{j+1,1}$ and $\mathrm{r}_{\mathrm{a}}\left(\widehat{\Gamma}_{j+1,1}\right)=\mathcal{O}(\eta)$, roughly speaking, $\widetilde{\Gamma}_{j+1,1}$ is nearly a vertical segment from the bottom to the top. Define $\Gamma_{j+1}^{1}$ be the component of $\widetilde{\Gamma}_{j+1,1}$ such that

$$
\max _{\mathbf{p} \in \Gamma_{j+1}^{1}} x_{2}(\mathbf{p})=\frac{1}{2}, \quad \min _{\mathbf{p} \in \Gamma_{j+1}^{1}} x_{2}(\mathbf{p})=\mathcal{O}\left(\tau_{0}\right), \quad \mathrm{r}_{\mathrm{a}}\left(\Gamma_{j+1}^{1}\right)=\mathcal{O}(\eta) .
$$

If $T\left(\Gamma_{j+1}^{1}\right) \cap\left(\left\{x_{1}=x_{2}\right\} \cup\left\{x_{1}+x_{2}=1\right\}\right) \neq \varnothing$, then the proof is completed. Thus assume the intersection set is empty. Then it is necessary that

$$
\max _{\mathbf{p} \in T\left(\Gamma_{j+1}^{1}\right)} x_{1}(\mathbf{p})=\mathcal{O}\left(\eta+\tau_{0}\right) .
$$

Otherwise, $\max _{T\left(\Gamma_{j+1}^{i_{0}}\right)} x_{1}(\mathbf{p}) \gg \eta+\tau_{0}$. Note that $T\left(\Gamma_{j+1}^{i_{0}}\right)$ is nearly a segment. Let $\mathbf{p}_{1}, \mathbf{p}_{2}$ be two end points of $T\left(\Gamma_{j+1}^{i_{0}}\right)$ such that $x_{2}\left(\mathbf{p}_{1}\right) \geq 1-\mathcal{O}\left(\tau_{0}\right)-\mathcal{O}(\eta)$ and $x_{2}\left(\mathbf{p}_{2}\right)=\mathcal{O}\left(\tau_{0}\right)$. Again from the fact that $T\left(\Gamma_{j+1}^{i_{0}}\right)$ is nearly a segment, we have either $x_{1}\left(\mathbf{p}_{1}\right) \gg \eta+\tau_{0}$ or $x_{1}\left(\mathbf{p}_{2}\right) \gg \eta+\tau_{0}$. For the former case, we have $x_{1}\left(\mathbf{p}_{1}\right)+x_{2}\left(\mathbf{p}_{1}\right)-1 \gg \eta+\tau_{0}-\tau_{0}-\eta=0$, while

$$
x_{1}\left(\mathbf{p}_{2}\right)+x_{2}\left(\mathbf{p}_{2}\right) \leq \frac{1}{2}+\mathcal{O}(\tau) \leq 1,
$$

which implies the intersection between $T\left(\Gamma_{j+1}^{i_{0}}\right)$ and $x_{1}+x_{2}=1$ is nonempty. For the latter case, we have $x_{2}\left(\mathbf{p}_{2}\right)<x_{1}\left(\mathbf{p}_{2}\right)$, while $x_{2}\left(\mathbf{p}_{1}\right)>\frac{1}{2}>x_{1}\left(\mathbf{p}_{1}\right)$, which implies that $T\left(\Gamma_{j+1}^{i_{0}}\right)$ and $x_{1}=x_{2}$ is nonempty, which makes the assumption on the empty intersection impossible.

Define $\Gamma_{j+1}^{2}$ as before such that

$$
\max _{\mathbf{p} \in \Gamma_{j+1}^{2}} x_{2}(\mathbf{p})=\frac{1}{2}, \quad \min _{\mathbf{p} \in \Gamma_{j+1}^{2}} x_{2}(\mathbf{p})=\mathcal{O}\left(\tau_{0}\right), \quad \mathrm{r}_{\mathrm{a}}\left(\Gamma_{j+1}^{2}\right)=\mathcal{O}(\eta) .
$$


From (5.4), it holds that there is an $i_{0} \leq\left[\log _{(s-\eta)(1-c)} \frac{1}{100} / \tau_{2}\right]+1$, such that $\max _{\mathbf{p} \in \Gamma_{j+1}^{2}} x_{1}\left(T^{i_{0}}(\mathbf{p})\right) \in\left[\frac{1}{100}, \frac{1}{10}\right]$. In fact, since $x_{1}(\mathbf{p}) \geq \tau_{2}$, from the argument above, we have $x_{1}\left(T^{i}(\mathbf{p})\right) \geq(s-\eta)(1-c) x_{1}\left(T^{i-1}(\mathbf{p})\right)$ only if $x_{1}\left(T^{i-1}(\mathbf{p})\right) \leq \frac{1}{100}$, which justifies the definition of $i_{0}$. In a word, we obtain a simple curve $\Gamma_{j+1}^{i_{0}}$ satisfying

$$
\begin{array}{ll}
\max _{\mathbf{p} \in \Gamma_{j+1}^{i_{0}}} x_{2}(T(\mathbf{p}))=1-\mathcal{O}\left(\tau_{0}\right), & \min _{\mathbf{p} \in \Gamma_{j+1}^{i_{0}}} x_{2}(T(\mathbf{p}))=\mathcal{O}\left(\tau_{0}\right), \\
\max _{\mathbf{p} \in \Gamma_{j+1}^{i_{0}}} x_{1}(T(\mathbf{p})) \in\left[\frac{1}{100}, \frac{1}{10}\right], & \mathrm{r}_{\mathrm{a}}\left(T\left(\Gamma_{j+1}^{i_{0}}\right)\right)=\mathcal{O}(\eta) .
\end{array}
$$

By the same argument above, we obtain $T\left(\Gamma_{j+1}^{i_{0}}\right) \cap\left(\left\{x_{1}=x_{2}\right\} \cup\left\{x_{1}+x_{2}=1\right\}\right) \neq$ $\varnothing$. We can end the proof of Lemma 5.3 similarly as before.

iii. $\widehat{\Gamma}_{j+1,1} \cap\left(\left\{x_{1}=\frac{1}{2}\right\}\right.$ (or $\widehat{\Gamma}_{j+1,1} \cap\left(\left\{x_{2}=\frac{1}{2}\right\}\right)$ includes two or more points, or $\widehat{\Gamma}_{j+1,2} \cap$ $\left(\left\{x_{1}=\frac{1}{2}\right\}\right.$ (or $\widehat{\Gamma}_{j+1,2} \cap\left(\left\{x_{2}=\frac{1}{2}\right\}\right)$ includes two or more points. It can be reduced to Case $4-(b)$-i by the same argument as in Case 2 , we omit it here.

We complete the proof.

Proof of (1.3a) in Theorem 1.3. From Lemma 5.1 and 5.3, we obtained that there exists $c_{6}>$ 0 such that for any segment $\Gamma_{0}$ in some small square with a slope 1 , there exists disjoint segments $\Gamma_{1}, \Gamma_{2}, \cdots$, such that for each $i$ there exists $l(i)$ such that $T^{l(i)}\left(\Gamma_{i}\right) \subset G_{\epsilon}$ and $\sum_{i} \mathrm{M}\left(\Gamma_{i}\right) \geq c_{6} \mathrm{M}\left(\Gamma_{0}\right)$. From the arbitrariness of $\Gamma_{0}$ and Fubini's Theorem, we obtain that for almost every initial point $\mathbf{p} \in[0,1]^{2}$, there exists $l(\mathbf{p})$ such that $T^{l(\mathbf{p})}(\mathbf{p}) \in G_{\epsilon}$. This completes the proof for (1.3a).

The disordered part can be obtained by a series of lemmas. First we prove a partial result as follows.

Lemma 5.4. There exists a subset $B_{0}$ of $G_{\gamma_{0}}$ with a positive measure such that for each point $\mathbf{p} \in B_{0}$, there exists a finite time $l=l(\mathbf{p})$ such that $T^{l}(\mathbf{p}) \in B_{\gamma_{0}}$.

Proof. Let $G_{1}=\left\{\mathbf{p} \in G_{\gamma_{0}} \mid x_{1}, x_{2} \leq \frac{1}{2}\right\}, G_{2}=\left\{\mathbf{p} \in G_{\gamma_{0}} \mid x_{1}, x_{2} \geq \frac{1}{2}\right\}, G_{3}=\left\{\mathbf{p} \in G_{\gamma_{0}} \mid x_{1} \leq\right.$ $\left.\frac{1}{2}, x_{2} \geq \frac{1}{2}\right\}, G_{4}=\left\{\mathbf{p} \in G_{\gamma_{0}} \mid x_{1} \geq \frac{1}{2}, x_{2} \leq \frac{1}{2}\right\}$ and define $\widetilde{G}_{\gamma_{0}}=G_{3} \cup G_{4}$. Let $\widetilde{G}_{-1}=$ $T^{-1}\left(\widetilde{G}_{\gamma_{0}}\right) \cap G_{\gamma_{0}}, \widetilde{G}_{-2}=T^{-1}\left(\widetilde{G}_{-1}\right) \cap G_{\gamma_{0}}, \cdots, \widetilde{G}_{-(k+1)}=T^{-1}\left(\widetilde{G}_{-k}\right) \cap G_{\gamma_{0}}$. Denote $G_{0}=$ $\cup_{k=0}^{\infty} \widetilde{G}_{-k}$.

Now, we define $B_{0} \equiv G_{\gamma_{0}} \backslash G_{0}$. Then for each point $\mathbf{p} \in B_{0}$, there exists a finite time $l=l(\mathbf{p})$ such that $T^{l}(\mathbf{p}) \in B_{\gamma_{0}}$. In fact, from the definition of the set $G_{0}$ and the map $T$, for each point $\mathbf{p} \in B_{0}$ satisfying $T^{i-1}(\mathbf{p}) \in G_{\gamma_{0}}$, it holds that $\left|x_{1}(i)-x_{2}(i)\right| \geq(1-2 c)(2-$ $\eta)\left|x_{1}(i-1)-x_{2}(i-1)\right|$, where $\left(x_{1}(j), x_{2}(j)\right)=T^{j}(\mathbf{p})$ for $j=i-1$, $i$. Hence, the fact that $(1-2 c)(2-\eta)>1$ with $c, \eta$ small implies that there exists some $l$ such that $T^{l}(\mathbf{p})$ is out of the region $G_{\gamma_{0}}$, i.e., . enter into $B_{\gamma_{0}}$. 
Thus it is sufficient to prove that $\mathrm{M}\left(G_{0}\right)<\mathrm{M}\left(G_{\gamma_{0}}\right)$. Since the diameter of $\widetilde{G}_{\gamma_{0}}$ is small for small $\gamma_{0}$, from the expansibility of $T$, we obtain that each component $S$ of $T^{-i}\left(\widetilde{G}_{\gamma_{0}}\right)$ possesses a small diameter for $i>0$. Thus, we have that

$$
\begin{array}{ll}
\text { Case (i). } \quad \#^{C}\left(T^{-1}(S) \cap G_{\gamma_{0}}\right)=2 & \text { if } \operatorname{dist}(S,(1,1))>3 \gamma_{0}, \\
\text { Case (ii). } \quad \#^{C}\left(T^{-1}(S) \cap G_{\gamma_{0}}\right) \leq 4 & \text { if } \operatorname{dist}(S,(1,1)) \leq 3 \gamma_{0},
\end{array}
$$

where $\#^{C}(S)$ denotes the number of components for the set $S$.

On the other hand, the expansibility of $T$ implies that the measure of each component of $T^{-1}(S)$ is less than $\left((1-2 c)(2-\eta)^{2}\right)^{-1} \mathrm{M}(S)$. Thus for case (i) we have that $\mathrm{M}\left(T^{-1}(S)\right) \leq 2\left((1-2 c)(2-\eta)^{2}\right)^{-1} \mathrm{M}(S) \equiv(2 \hat{\lambda})^{-1} \mathrm{M}(S)$ and for case (ii) we have that $\mathrm{M}\left(T^{-1}(S)\right) \leq 4\left((1-2 c)(2-\eta)^{2}\right)^{-1} \mathrm{M}(S) \equiv \hat{\lambda}^{-1} \mathrm{M}(S)$ with $1-\mathcal{O}(c+\eta) \leq \hat{\lambda} \leq 1$.

Obviously, since $\gamma_{0}$ is small, we have that among $\{i, i+1, \cdots, i+10\}$, there is at most one number $j$ such that $\operatorname{dist}\left(T^{-j}(S),(1,1)\right) \leq 3 \gamma_{0}$. Thus it is not difficult to see that for $k=10 l+j$ with $1 \leq j<10$, it holds that $\mathrm{M}\left(\widetilde{G}_{-k}\right) \leq b_{k} \mathrm{M}\left(\widetilde{G}_{\gamma_{0}}\right)$ with $b_{k}=$ $\left((2 \hat{\lambda})^{-9} \hat{\lambda}^{-1}\right)^{l}(2 \hat{\lambda})^{-(j-1)} \hat{\lambda}^{-1}$. Let $b_{0}=1$. For small $c$ and $\eta$ we can easily see that $\sum_{k=0}^{\infty} b_{k} \leq$ 4. Hence we have

$$
\mathrm{M}\left(\cup_{k=0}^{\infty} \widetilde{G}_{-k}\right) \leq \sum_{k=0}^{\infty} b_{k} \mathrm{M}\left(\widetilde{G}_{\gamma_{0}}\right) \leq 4 \mathrm{M}\left(\widetilde{G}_{\gamma_{0}}\right) \leq 4 \gamma_{0} \mathrm{M}\left(G_{\gamma_{0}}\right)<\mathrm{M}\left(G_{\gamma_{0}}\right) .
$$

This completes the proof of the lemma.

The disordered part (1.3b) can be easily obtained from the following corollary.

Corollary 5.1. For almost each point $\mathbf{p}$ in $G_{\gamma_{0}}$, there exists a finite time $l=l(\mathbf{p})$ such that $T^{l}(\mathbf{p}) \in B_{\gamma_{0}} \cup B_{0}$, where $B_{0}$ is defined as in Lemma 5.4.

Proof. Assume the conclusion is not true. Then there exists a set $S \subset G_{\gamma_{0}}$ with a positive measure such that for each $i$ it holds that $T^{i}(S) \cap\left(B_{\gamma_{0}} \cup B_{0}\right)=\varnothing$. We will prove that there exists a subset $S_{0} \subset S$ with a positive measure and $l=l\left(S_{0}\right)$ such that $T^{l}\left(S_{0}\right) \subset B_{\gamma_{0}} \cup B_{0}$. From the contradiction, we end the proof.

For this purpose, we claim that there exists a subset $\widetilde{S_{0}} \subset S$ and $l \in \mathbb{N}$ such that $\mathrm{M}\left(T^{l}\left(\widetilde{S_{0}}\right)\right) \geq \delta_{1} \mathrm{M}\left(G_{\gamma_{0}}\right)$.

Then the Corollary can be obtained from the claim. In fact, since $T^{l}\left(\widetilde{S_{0}}\right) \subset T^{l}(S) \subset G_{\gamma_{0}}$ and $B_{0}=G_{\gamma_{0}} \backslash G_{0}$, we have $T^{l}\left(\widetilde{S}_{0}\right) \backslash B_{0} \subset G_{0}$, where $G_{0}$ is defined as in the proof of Lemma 5.4. Note that $T$ is a diffeomorphism on each small square, thus the image of each measurable set under $T$ is still measurable. Thus

$$
\mathrm{M}\left(T^{l}\left(\widetilde{S_{0}}\right) \cap B_{0}\right)=\mathrm{M}\left(T^{l}\left(\widetilde{S_{0}}\right)\right)-\mathrm{M}\left(T^{l}\left(\widetilde{S_{0}}\right) \backslash B_{0}\right) \geq \mathrm{M}\left(T^{l}\left(\widetilde{S_{0}}\right)\right)-\mathrm{M}\left(G_{0}\right) .
$$

Then from the claim and (5.5), we have

$$
\frac{\mathrm{M}\left(T^{l}\left(\widetilde{S_{0}}\right) \cap B_{0}\right)}{\mathrm{M}\left(T^{l}\left(\widetilde{S_{0}}\right)\right)} \geq \frac{\mathrm{M}\left(T^{l}\left(\widetilde{S_{0}}\right)\right)-\mathrm{M}\left(G_{0}\right)}{\mathrm{M}\left(T^{l}\left(\widetilde{S_{0}}\right)\right)} \geq 1-\frac{4 \gamma_{0}}{\delta_{1}} \geq \frac{1}{2} \text {, since } \gamma_{0} \ll \delta_{1} \text {. }
$$


Define $S_{0} \subset \widetilde{S_{0}}$ such that $T^{l}\left(S_{0}\right)=T^{l}\left(\widetilde{S_{0}}\right) \cap B_{0} \subset B_{0}$. Then $T^{l}\left(S_{0}\right)$ has a positive measure, which implies the measure of $S_{0}$ is positive. Thus the conclusion is obtained.

Next we prove the existence of $\widetilde{S_{0}}$. From the definition of $G_{i}, i=1,2,3,4$ and the number of components of a set in $[0,1]^{2}$, we have that for any subset $S$ of $G_{3}$ or $G_{4}, T^{j}(S)$ has only one component for each $j=1,2, \cdots, 10$. Recall that for any subset $S$ of $G_{1}$ or $G_{2}$, $T(S)$ has at most 4 components (see Fig. 4).

Without loss of generality, we assume that $S \subset G_{1}$. Then $T(S)$ has at most 4 components among which 2 components, say $S_{1}$ and $S_{2}$ lie in $G_{1}$ and $G_{2}$, respectively, and $S_{3}$ and $S_{4}$ lie in $G_{3}$ and $G_{4}$. Subsequently, $T\left(S_{1}\right)$ or $T\left(S_{2}\right)$ have at most 4 components denoted by $S_{i, j}, i=1,2, j=1,2,3,4$ in a similar way, while $T\left(S_{3}\right)$ or $T\left(S_{4}\right)$ has only one components denoted by $S_{i, 1}, i=3,4$. Hence, all $T\left(S_{1, i}\right), i=1,2,3,4$ totally have 10 components. Moreover, among them there are six components, that is $S_{i, 1}, i=3,4$ together with $S_{i, j}$, $i=1,2, j=3,4$, satisfy that the image of each of them has only one component.

By induction, we can prove that for $i \leq 10$, it holds that the sum of all components for $T^{i}(S)$ is $2^{i+1}+2^{i}-2$. In particular, the sum of all components for $T^{3}(S)$ is 22.

On the other hand, $\mathrm{M}\left(T^{3}(S)\right) \geq(4(1-2 c-2 \eta))^{3} \mathrm{M}(S)$. When $c, \eta$ are small, we have that $(1-2 c)(2-\eta)^{2}>(22)^{1 / 3}$. Applying Corollary 2.1 by setting $a=22, m_{0}=3$, $E_{-}(c)=(1-2 c)(2-\eta)^{2}, E_{+}(c)=(1-2 c)(2+\eta)^{2}, \delta_{1}=2^{-16}, \gamma_{0}=2^{-20}$ and $D=G_{\gamma_{0}}$, we obtain the existence of $\widetilde{S_{0}}$. The proof is completed.

Rules:

1. $T^{\mathrm{j}}(\mathrm{S})$ has only one branch when $\mathrm{S}$ is from $\mathrm{G}_{3}$ and $\mathrm{G}_{4}, \forall 1 \leq \mathrm{j} \leq 10$;

2. $T^{j}(S)$ has less than five branches when $S$ is from $G_{1}$ and $G_{2}, \forall 1 \leq j \leq 10$.

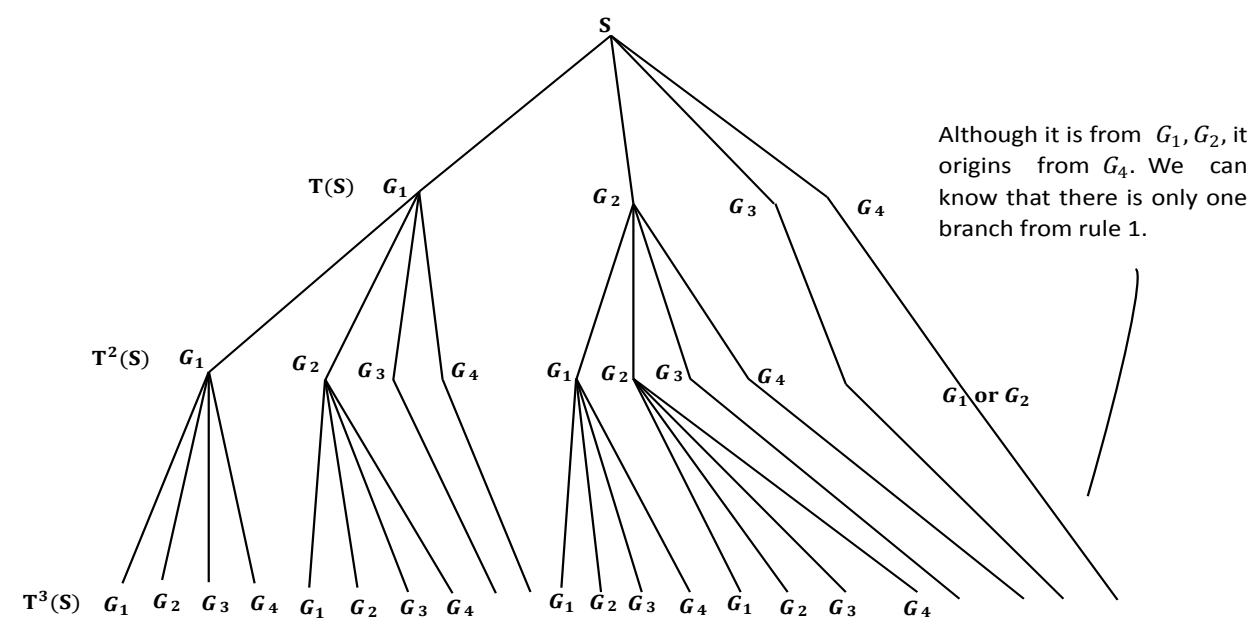

Figure 4: Evolution on components of $T^{j}(S), j=1,2,3$. 


\section{The higher dimensional case}

In this section, we will prove Theorem 1.4 for coupled tent map lattices with multi-node.

The proof for the ordered part in the multi-node case is quite different from for twonodes case. The observation is as follows.

Recall that the phase space $[0,1]^{m}=\cup D_{J}$, where $m$ is the dimension of the phase space and $D_{J}$ are $2^{m}$ small hypercubes in the phase space divided by the planes $x_{i}=\frac{1}{2}, 1 \leq i \leq$ $m$. For each convex $\Omega$ in some $D_{J_{0}}$, we have that $\mathrm{M}(T(\Omega)) \approx 2^{m} \mathrm{M}(\Omega)$. Clearly $T(\Omega)$ will either has an intersection with each of $2^{m}$ small hypercubes $D_{J}$ simultaneously, or there exists at least one hypercube which has no intersection point with $T(\Omega)$. Note that all $T(\Omega) \cap D_{J}$ are still convex. Once the former case occurs, from the convexity we can prove that $T(\Omega)$ has an intersection with the diagonal $D_{\text {syn, }}$, which again by convexity implies the existence of a set of 'good' points occupying a fixed ratio in $\Omega$. Otherwise, suppose the latter case occurs in each iteration step. Then in each iteration step, averagely it holds that $\mathrm{M}\left(T(\Omega) \cap D_{J}\right) \geq c \mathrm{M}(\Omega)$ with $c \approx 2^{m} /\left(2^{m}-1\right)>1$ for each $J$. Consequently, the measure for most components of $T^{k}(\Omega)$ will keep increasing until it is of constant order as $k$ increases. Thus we also obtain the existence of a set of 'good' points occupying a fixed ratio in $\Omega$ by convexity.

To prove the ordered part (1.3a), we first have the following result.

Lemma 6.1. If $\Omega$ is a convex set in one of the $2^{m}$ small hypercubes $D_{J}$ and $T(\Omega)$ has an intersection with each small hypercube simultaneously. Then the center point $x_{1}=x_{2}=\cdots=x_{m}=\frac{1}{2}$ lies in $T(\Omega)$. Moreover, there exists a fixed number $c_{0}>0$ such that for any $\epsilon>0$, we have that $\mathrm{M}\left(T(\Omega) \cap G_{\epsilon}\right) / \mathrm{M}(T(\Omega)) \geq c_{0} \epsilon^{m}$, where

$$
G_{\epsilon}=\left\{\mathbf{p} \in[0,1]^{m} \mid \operatorname{dist}\left(\mathbf{p}, D_{\text {syn }}\right) \leq \epsilon\right\} .
$$

Proof. We prove the first conclusion by induction. For $m=2$, let

$$
D_{i j}=\left\{\left(x_{1}, x_{2}\right) \in[0,1]^{2} \mid \frac{(i-1)}{2} \leq x_{1} \leq \frac{i}{2}, \frac{(j-1)}{2} \leq x_{2} \leq \frac{j}{2}\right\},
$$

$i, j=1,2$, be all the small hypercubes. From the condition, we have that for each pair $(i, j)$, there exists a point $\mathbf{p}_{i j} \in D_{i j} \cap T(\Omega)$. Then from the convexity, we have that the convex hull determined by these points is a subset of $T(\Omega)$ and the point $\left(\frac{1}{2}, \frac{1}{2}\right)$ is in it. Thus the first conclusion is proved.

Assume the first conclusion holds true for $k=2, \ldots, m-1$. For the case $k=m$, consider $\widehat{D}_{\frac{1}{2}}=\left\{\left(x_{1}, \ldots, x_{m}\right) \in[0,1]^{m} \mid x_{m}=\frac{1}{2}\right\}$. Obviously it consists of $2^{m-1}$ small $(m-1)$-dimensional hypercubes determined by the planes $x_{i}=\frac{1}{2}, i=1, \ldots, m-1$. We have that $\widehat{D}_{\frac{1}{2}} \cap T(\Omega)$ is nonempty and convex by the convexity of $\Omega$, since in $T(\Omega)$ there exist both points with $x_{m}<\frac{1}{2}$ and the ones with $x_{m}>\frac{1}{2}$. Furthermore, the condition implies that the intersection between $T(\Omega)$ and each small hypercubes of $\widehat{D}_{\frac{1}{2}}$ is nonempty. 
Thus applying inductive assumption for $m-1$ on $\widehat{D}_{\frac{1}{2}} \cap T(\Omega)$ and $\widehat{D}_{\frac{1}{2}}$, we have that the point $\left(\frac{1}{2}, \ldots, \frac{1}{2}\right) \in \widehat{D}_{\frac{1}{2}} \cap T(\Omega)$, which leads to the first conclusion.

For the second conclusion, let $S_{\epsilon}$ be the cylinder $\left\{\mathbf{p} \in[0,1]^{m} \mid \operatorname{dist}\left(\mathbf{p}, D_{\text {syn }}\right)=\epsilon\right\}$ whose axis is the diagonal of the phase space.

Define $\widehat{S}_{\epsilon}=S_{\epsilon} \cap T(\Omega)$ and let $\Gamma$ be the point set of the union of all lines connecting $\widehat{S}_{\epsilon}$ and the point $\mathbf{p}_{0}=\left(\frac{1}{2}, \ldots, \frac{1}{2}\right)$. From the first conclusion it holds that $\mathbf{p}_{0} \in T(\Omega)$. Hence, if $\widehat{S}_{\epsilon}$ is empty, then the convexity of $T(\Omega)$ leads that $T(\Omega) \subset G_{\epsilon}$ and the proof is complete. Thus we assume that $\widehat{S}_{\epsilon}$ is nonempty. Obviously, $T(\Omega) \backslash(\Gamma \cap T(\Omega)) \subset T(\Omega) \cap G_{\epsilon}$. Thus for our purpose, we only need to analyze $\Gamma \cap T(\Omega)$. Again by the convexity we have that $\Omega_{\epsilon} \equiv \Gamma \cap G_{\epsilon} \subset T(\Omega)$, which further implies

$$
\Omega_{\epsilon}=(\Gamma \cap T(\Omega)) \cap G_{\epsilon} .
$$

To prove the second conclusion, it is sufficient to estimate $\mathrm{M}\left(\Omega_{\epsilon}\right) / \mathrm{M}(\Gamma \cap T(\Omega))$. Note that $\Gamma \cap T(\Omega) \subset \Gamma \cap[0,1]^{m}$.

For each line $L \in \Gamma$, we can easily see that the length $L \cap[0,1]^{m}$ is less than $\sqrt{m}$, while the length of $L \cap G_{\epsilon}$ is larger than $\epsilon$. For the definition of $\Omega_{\epsilon}$ and (6.1), we thus have that

$$
\mathrm{M}\left((\Gamma \cap T(\Omega)) \cap G_{\epsilon}\right)=\mathrm{M}\left(\Omega_{\epsilon}\right) \geq\left(\frac{\epsilon}{\sqrt{m}}\right)^{m} \mathrm{M}\left(\Gamma \cap[0,1]^{m}\right) \geq\left(\frac{\epsilon}{\sqrt{m}}\right)^{m} \mathrm{M}(\Gamma \cap T(\Omega)) .
$$

Hence we complete the proof of the lemma by setting $c_{0}=m^{-m / 2}$.

Applying Lemma 6.1, we obtain the following result.

Proposition 6.1. There exists a constant $c_{1}>0$ such that for any convex set $\Omega$ with a volume less than $10^{-m}$, there exist disjoint convexities $\Omega_{i} \subset \Omega, i=1,2, \ldots$, and a set $\Omega_{0} \subset \Omega$ which is a union of finite convexities such that (i) for any $i \geq 1$, there exists a $l(i)$ such that $T^{l(i)}\left(\Omega_{i}\right)$ is a component of $T^{l(i)}(\Omega)$ and $\mathrm{M}\left(T^{l(i)}\left(\Omega_{i}\right)\right) \geq \delta_{1}$; (ii) for any point $\mathbf{p} \in \Omega_{0}$, there exists an $l(\mathbf{p})$ such that $T^{l(\mathbf{p})}(\mathbf{p}) \in G_{\epsilon} ;$ (iii) $\mathrm{M}\left(\cup_{i \geq 0} \Omega_{i}\right) \geq c_{1} \mathrm{M}(\Omega)$.

Proof. From Lemma 6.1, without loss of generality we assume that there exists at least one small hypercube which has no intersection point with $T(\Omega)$ for any convex $\Omega$ in some small hypercube. Thus $T(\Omega)$ has at most $2^{m}-1$ components, denoted by $\Omega_{i}, i=$ $1, \cdots, 2^{m}-1$ (some of them may be empty).

On the other hand,

$$
|\operatorname{det}(J T(\mathbf{p}))|=2^{m}|\operatorname{det}(I+c A)| \equiv 2^{m}(1-F(A, c)),
$$

where $F(A, c)$ depends on $A^{\top}=A \in \mathbb{R}^{m \times m}$ with $A \mathbf{e}=0$ and the coupling coefficient $c$ satisfying $F(A, c) \rightarrow 0$ as $c \rightarrow 0$. Then we have

$$
|\operatorname{det}(J T(\mathbf{p}))|>2^{m}-1 \text { for small } c .
$$

Thus from Iteration Lemma 2.1 and Corollary 2.1 with $m_{0}=1, a=2^{m}-1$ and $E_{-}(c)=$ $2^{m}(1-F(A, c))$, we complete the proof of this proposition. 
The ordered part (1.3a) for the case of multi-node can be reduced to the following result.

Proposition 6.2. For any $\epsilon>0$, there exists a fixed number $c_{2}>0$ depending on $\epsilon$ such that for any convex set $\Omega_{0}$ in some small hypercube with $\mathrm{M}\left(\Omega_{0}\right) \geq \delta_{1}$, there exist disjoint convexities $\Omega_{i} \subset \Omega_{0}, i=1,2, \ldots$, such that (i) for any $i \geq 1$, there exists an $l(i)$ such that $T^{l(i)}\left(\Omega_{i}\right) \subset G_{\epsilon}$ and (ii) $\mathrm{M}\left(\cup_{i \geq 1} \Omega_{i}\right) \geq c_{2} \mathrm{M}\left(\Omega_{0}\right)$.

Proof. If $T\left(\Omega_{0}\right) \cap D_{J} \neq \varnothing$ for each small hypercube $D_{J}$ of $D$, then the conclusion follows from Lemma 6.1.

Otherwise, there exist at most $2^{m}-1$ small hypercubes of $[0,1]^{m}$ such that $T\left(\Omega_{0}\right) \cap$ $D_{J} \neq \varnothing$. Recall that $\mathrm{M}\left(T\left(\Omega_{0}\right)\right) \geq 2^{m}(1-F(A, c)) \mathrm{M}\left(\Omega_{0}\right)$. Hence there exists some $D_{J}$ such that the convex set $\Omega_{1}=T\left(\Omega_{0}\right) \cap D_{\text {J }}$ possesses a volume larger than

$$
\left(2^{m} /\left(2^{m}-1\right)\right)(1-F(A, c)) \mathrm{M}\left(\Omega_{0}\right) .
$$

Similarly, assume that there exists at most $2^{m}-1$ small hypercubes of $[0,1]^{m}$ such that $T\left(\Omega_{1}\right) \cap D_{J} \neq \varnothing$. Then repeating the above argument, we obtain a convex set $\Omega_{i+1}=$ $T\left(\Omega_{i}\right) \cap D_{J}$ for some $D_{J}$ such that

$$
\begin{aligned}
\mathrm{M}\left(\Omega_{i+1}\right) & \geq 2^{m} /\left(2^{m}-1\right)(1-F(A, c)) \mathrm{M}\left(\Omega_{i}\right) \\
& \geq\left(2^{m} /\left(2^{m}-1\right)(1-F(A, c))\right)^{i+1} \mathrm{M}\left(\Omega_{0}\right)
\end{aligned}
$$

for any $i$. Since $F(A, c) \rightarrow 0$ and $\mathrm{M}\left(\Omega_{0}\right) \geq \delta_{1}$, we have that

$$
\left(2^{m} /\left(2^{m}-1\right)(1-F(A, c))\right)^{j} \mathrm{M}\left(\Omega_{0}\right)>1
$$

for small $c$ and some fixed $j=j\left(\delta_{1}\right)$. Hence there exists some $i<j$ such that $T\left(\Omega_{i}\right) \cap D_{J} \neq$ $\varnothing$ for small hypercubes $D_{J}$ of $[0,1]^{m}$, which leads to the conclusion with

$$
c_{2}>c_{0} \mathrm{M}\left(G_{\epsilon}\right) 2^{-m j} \geq c_{0} \epsilon^{m-1} 2^{-m j},
$$

where $c_{0}$ is defined in Lemma 6.1.

Similar to the proof of Theorem 3.1, we can easily obtain (1.3a) from Proposition 6.2. Thus we omit the details.

The disordered part for the multi-node case is similar to the two-node case. Let $\gamma_{0}=$ $\min \left\{2^{-20}, 2^{-m-4}\right\}$. First we prove that

Lemma 6.2. There exists a subset $B_{0}$ of $G_{\gamma_{0}}$ with a positive measure such that for each point $\mathbf{p} \in B_{0}$, there exists a finite time $l=l(\mathbf{p})$ such that $T^{l}(\mathbf{p}) \in B_{\gamma_{0}}$.

Proof. Let

$$
\begin{aligned}
& D_{J_{1}}=\left\{\left(x_{1}, \ldots, x_{n}\right) \in D \mid 0 \leq x_{i} \leq \frac{1}{2}, \text { for all } i\right\}, \\
& D_{J_{2}}=\left\{\left(x_{1}, \ldots, x_{n}\right) \in D \mid \frac{1}{2} \leq x_{i} \leq 1, \text { for all } i\right\} .
\end{aligned}
$$


Define $\widetilde{G}_{\gamma_{0}}=G_{\gamma_{0}} \backslash\left(D_{J_{1}} \cup D_{J_{2}}\right)$. Let

$$
\begin{aligned}
& \widetilde{G}_{-1}=T^{-1}\left(\widetilde{G}_{\gamma_{0}}\right) \cap G_{\gamma_{0}}, \quad \widetilde{G}_{-2}=T^{-1}\left(\widetilde{G}_{-1}\right) \cap G_{\gamma_{0}}, \ldots, \\
& \widetilde{G}_{-(l+1)}=T^{-1}\left(\widetilde{G}_{-l}\right) \cap G_{\gamma_{0}} .
\end{aligned}
$$

Denote $G_{0}=\cup_{l=0}^{\infty} \widetilde{G}_{-l}$. We claim that for each point $\mathbf{p} \in B_{0}=G_{\gamma_{0}} \backslash G_{0}$, there exists some $l$ such that $T^{l}(\mathbf{p})$ is out of the region $G_{\gamma_{0}}$, i.e., enters into $B_{\gamma_{0}}$. Thus for our purpose it is sufficient to prove that $\mathrm{M}\left(G_{0}\right)<\mathrm{M}\left(G_{\gamma_{0}}\right)$. From the definition of the set $G_{0}$ and the map $T$, for each point $\mathbf{p} \in B_{0}$, it holds that $T^{i}(\mathbf{p}) \notin \widetilde{G}_{\gamma_{0}}$ for any $i$. By the expansivity of $T$, we need to prove that if $T^{i-1}(\mathbf{p}) \in G_{\gamma_{0}}$, then

$$
\operatorname{dist}\left(T^{i}(\mathbf{p}), D_{\text {syn }}\right) \geq 2(1-\widehat{F}(A, c)) \operatorname{dist}\left(T^{i-1}(\mathbf{p}), D_{\text {syn }}\right),
$$

where $\widehat{F}(A, c) \rightarrow 0$ (see below for definition), as $c \rightarrow 0$.

In fact, for $\mathbf{x}=\left[x_{1}, \ldots, x_{m}\right]^{\top}$, we have

$$
\left(\operatorname{dist}\left(\mathbf{x}, D_{\text {syn }}\right)\right)^{2}=(2 m)^{-1} \sum_{i \neq j}\left(x_{i}-x_{j}\right)^{2}=\sum_{i=1}^{m} x_{i}^{2}-m^{-1}\left(\sum_{i=1}^{m} x_{i}\right)^{2}=\mathbf{x}^{T}\left(I-m^{-1} E_{0}\right) \mathbf{x},
$$

where $E_{0}=\mathbf{e e}^{\top}$ with $\mathbf{e}=[1, \cdots, 1]^{T}$. Then it holds that

$$
\begin{aligned}
& \left(\operatorname{dist}\left(T(\mathbf{x}), D_{\text {syn }}\right)\right)^{2}=(T(\mathbf{x}))^{T}\left(I-m^{-1} E_{0}\right) T(\mathbf{x}) \\
= & 4 \mathbf{x}^{T}(I+c A)^{T}\left(I-m^{-1} E_{0}\right)(I+c A) \mathbf{x} .
\end{aligned}
$$

From the condition of $A$ it follows that $A E_{0}=E_{0} A=0$. It implies that

$$
(I+c A)^{T}\left(I-m^{-1} E_{0}\right)(I+c A)=I-m^{-1} E_{0}+2 c A+c^{2} A^{2} .
$$

It leads that

$$
\begin{aligned}
\left(\operatorname{dist}\left(T(\mathbf{x}), D_{\text {syn }}\right)\right)^{2} & =4 \mathbf{x}^{T}\left(I-m^{-1} E_{0}+2 c A+c^{2} A^{2}\right) \mathbf{x} \\
& =4\left(\operatorname{dist}\left(\mathbf{x}, D_{\text {syn }}\right)\right)^{2}+4 \mathbf{x}^{T}\left(2 c A+c^{2} A^{2}\right) \mathbf{x} .
\end{aligned}
$$

Denote

$$
\overline{\mathbf{x}}=\frac{1}{m} \sum_{i=1}^{m} x_{i}
$$

Then we have

$$
\begin{aligned}
& \mathbf{x}^{T}\left(2 c A+c^{2} A^{2}\right) \mathbf{x} \\
= & (\overline{\mathbf{x}} \mathbf{e}+(\mathbf{x}-\overline{\mathbf{x}} \mathbf{e}))^{T}\left(2 c A+c^{2} A^{2}\right)(\overline{\mathbf{x}} \mathbf{e}+(\mathbf{x}-\overline{\mathbf{x}} \mathbf{e})) \\
= & (\overline{\mathbf{x}} \mathbf{e})^{T}\left(2 c A+c^{2} A^{2}\right)(\overline{\mathbf{x}} \mathbf{e})+2(\overline{\mathbf{x}} \mathbf{e})^{T}\left(2 c A+c^{2} A^{2}\right)(\mathbf{x}-\overline{\mathbf{x}} \mathbf{e}) \\
& \quad+(\mathbf{x}-\overline{\mathbf{x}} \mathbf{e})^{T}\left(2 c A+c^{2} A^{2}\right)(\mathbf{x}-\overline{\mathbf{x}} \mathbf{e}) .
\end{aligned}
$$


Again from the condition of $A$, we have

$$
(\overline{\mathbf{x}} \mathbf{e})^{T}\left(2 c A+c^{2} A^{2}\right)(\overline{\mathbf{x}} \mathbf{e})=0, \quad 2(\overline{\mathbf{x}} \mathbf{e})^{T}\left(2 c A+c^{2} A^{2}\right)=0 .
$$

On the other hand, it is easily seen that

$$
\left(\operatorname{dist}\left(\mathbf{x}, D_{\text {syn }}\right)\right)^{2}=\sum_{i=1}^{m}\left(x_{i}-\overline{\mathbf{x}}\right)^{2}=(\operatorname{dist}(\mathbf{x}, \overline{\mathbf{x}} \mathbf{e}))^{2} .
$$

Then we have that

$$
\left|\mathbf{x}^{T}\left(2 c A+c^{2} A^{2}\right) \mathbf{x}\right| \leq\left(2 c\|A\|+c^{2}\|A\|^{2}\right)\left(\operatorname{dist}\left(\mathbf{x}, D_{\text {syn }}\right)\right)^{2} .
$$

It follows that

$$
\begin{aligned}
& \left(\operatorname{dist}\left(T(\mathbf{x}), D_{\text {syn }}\right)\right)^{2} \geq 4\left(1-\left(2 c\|A\|+c^{2}\|A\|^{2}\right)\right)\left(\operatorname{dist}\left(\mathbf{x}, D_{\text {syn }}\right)\right)^{2} \\
\geq & \left(2\left(1-\left(2 c\|A\|+c^{2}\|A\|^{2}\right)^{\frac{1}{2}}\right)\right)^{2}\left(\operatorname{dist}\left(\mathbf{x}, D_{\text {syn }}\right)\right)^{2} \\
\equiv & (2(1-\widehat{F}(A, c)))^{2}\left(\operatorname{dist}\left(\mathbf{x}, D_{\text {syn }}\right)\right)^{2} .
\end{aligned}
$$

Thus we obtain the claim. Consequently there must exists $l$ such that $T^{l}(\mathbf{x}) \in B_{\gamma_{0}}$.

Next we estimate the measure of the set $G_{0}$. Since the diameter of $\widetilde{G}_{\gamma_{0}}$ is small for small $\gamma_{0}$, from the expansivity of $T$, we obtain that each component $S$ of $T^{-i}\left(\widetilde{G}_{\gamma_{0}}\right)$ possesses a small diameter for $i>0$. Thus we have that

$$
\begin{array}{ll}
\text { Case (i). } & \#^{C}\left(T^{-1}(S) \cap G_{\gamma_{0}}\right)=2 \quad \text { if } \operatorname{dist}(S,(1, \ldots, 1))>3 \gamma_{0}, \\
\text { Case (ii). } \quad \#^{C}\left(T^{-1}(S) \cap G_{\gamma_{0}}\right) \leq 2^{m} \quad \text { if } \operatorname{dist}(S,(1, \ldots, 1)) \leq 3 \gamma_{0},
\end{array}
$$

where $\#^{C}(S)$ denotes the number of connected components for a set $S$.

On the other hand, the expansibility of $T$ implies that the measure of each component of $T^{-1}(S)$ is less than $(2(1-\widehat{F}(A, c)))^{-m} \mathrm{M}(S)$. Thus for case (i) we have that $\left.\mathrm{M}\left(T^{-1}(S)\right) \leq 2^{-m+1}(1-\widehat{F}(A, c))\right)^{-m} \mathrm{M}(S)$ and for case (ii) we have that

$$
\mathrm{M}\left(T^{-1}(S)\right) \leq(1-\widehat{F}(A, c))^{-m} \mathrm{M}(S) .
$$

Obviously, since $\gamma_{0}$ is small, we have that there is at most one number $j$ in $\{i, i+$ $1, \cdots, i+10\}$ such that $\operatorname{dist}\left(T^{-j}(S),(1, \ldots, 1)\right) \leq 3 \gamma_{0}$. Thus it is not difficult to see that for $l=10 k+j$ with $1 \leq j<10$, it holds that $\mathrm{M}\left(\widetilde{\mathrm{G}}_{-l}\right) \leq d_{l} \mathrm{M}\left(\widetilde{\mathrm{G}}_{\gamma_{0}}\right)$ with $d_{l}=2^{(-m+1)(9 k+j-1)}(1-\widehat{F}(A, c))^{-m l}$. Let $d_{0}=1$. For small $c$ we can easily see that $\sum_{l=0}^{\infty} d_{l} \leq 4$. Hence if $0 \leq \gamma_{0}<\frac{1}{2^{m+3}}$ and $c$ is small, we have

$$
\begin{aligned}
\mathrm{M}\left(G_{0}\right) & \leq \mathrm{M}\left(\cup_{l=0}^{\infty} T^{-l}\left(\widetilde{G}_{\gamma_{0}}\right)\right) \leq \sum_{l=0}^{\infty} d_{l} \mathrm{M}\left(\widetilde{G}_{\gamma_{0}}\right) \leq 4 \mathrm{M}\left(\widetilde{G}_{\gamma_{0}}\right) \\
& \leq 4 \gamma_{0} \mathrm{M}\left(G_{\gamma_{0}}\right)<\frac{1}{2^{m+1}} \mathrm{M}\left(G_{\gamma_{0}}\right) .
\end{aligned}
$$

This completes the proof of the lemma. 
The disordered part (1.3b) for the case of multi-node can be easily obtained from the following corollary.

Corollary 6.1. For almost each point $\mathbf{p}$ in $G_{\gamma_{0}}$, there exists a finite time $l=l(\mathbf{p})$ such that $T^{l}(\mathbf{p}) \in B_{\gamma_{0}} \cap B_{0}$, where $B_{0}$ is defined as in Lemma 6.2.

Proof. Assume the conclusion is not true. Then there exists a set $S \subset G_{\gamma_{0}}$ with a positive measure such that for each $l$ it holds that $T^{l}(S) \cap\left(B_{\gamma_{0}} \cup B_{0}\right)=\varnothing$. We will prove that there exists a subset $S_{0} \subset S$ with a positive measure and $l=l\left(S_{0}\right)$ such that $T^{l}\left(S_{0}\right) \subset B_{\gamma_{0}} \cup B_{0}$. From the contradiction, we end the proof.

For this purpose, we claim that there exists a subset $\widetilde{S_{0}} \subset S$ and $l \in \mathbb{N}$ such that $\mathrm{M}\left(T^{l}\left(\widetilde{S_{0}}\right)\right) \geq \delta_{1} \mathrm{M}\left(G_{\gamma_{0}}\right)$.

In fact, from the claim and (6.2), we have that

$$
\frac{\mathrm{M}\left(T^{l}\left(\widetilde{S_{0}}\right) \cap B_{0}\right)}{\mathrm{M}\left(T^{l}\left(\widetilde{S_{0}}\right)\right)} \geq \frac{\mathrm{M}\left(T^{l}\left(\widetilde{S_{0}}\right)\right)-\mathrm{M}\left(G_{0}\right)}{\mathrm{M}\left(T^{l}\left(\widetilde{S_{0}}\right)\right)} \geq 1-\frac{4 \gamma_{0}}{\delta_{1}} \geq \frac{1}{2}
$$

Define $S_{0} \subset \widetilde{S_{0}}$ such that $T^{l}\left(S_{0}\right)=T^{l}\left(\widetilde{S_{0}}\right) \cap B_{0} \subset B_{0}$. Then $T^{l}\left(S_{0}\right)$ has a positive measure, which implies the measure of $S_{0}$ is positive. Thus the conclusion is obtained.

Next we prove the existence of $\widetilde{S_{0}}$. Let $G_{\gamma_{0}}=G_{1} \cup G_{2} \cup G_{3}$, where $G_{1}=G_{\gamma_{0}} \cap D_{J_{1}}$, $G_{2}=G_{\gamma_{0}} \cap D_{J_{2}}$ and $G_{3}=G_{\gamma_{0}} \backslash\left(G_{1} \cup G_{2}\right)$. It is clear that for a subset $S$ of any component of $G_{3}, T^{j}(S)$ has only one component for each $j=1, \ldots, 10$. Moreover, the image of any subset of $G_{1}$ or $G_{2}$ under $T$ has at most $2^{m}$ components.

Then for a subset $S$ of $G_{1}$ or $G_{2}, T(S)$ has at most $2^{m}$ components among which two ones lie in $G_{1} \cup G_{2}$ and the other $2^{m}-2$ ones lie in $G_{3}$. Subsequently, $T^{2}(S)$ has at most $\left(2^{m}-2\right)+2 \cdot 2^{m}$ components, among which 4 components lie in $G_{1} \cup G_{2}$ and $3\left(2^{m}-2\right)$ others lie in $G_{3}$. Similarly, we have that $T^{3}(S)$ has at most $\left(2^{3}-1\right)\left(2^{m}-2\right)+2^{3}$ components, among which $\left(2^{3}-1\right)\left(2^{m}-2\right)$ components lie in $G_{3}$ and $2^{3}$ others lie in $G_{1} \cup G_{2}$.

On the other hand,

$$
\mathrm{M}\left(T^{3}(S)\right) \geq 2^{3 m}(1-F(A, c))^{3} \mathrm{M}(S),
$$

where $F(A, c)$ is defined in Proposition 6.1. Obviously,

$$
2^{3 m}(1-F(A, c))^{3 m}>\left(2^{3}-1\right)\left(2^{m}-2\right)+2^{3}
$$

for $m \geq 2$ and small $c$. Thus from Corollary 2.1, we obtain the existence of $\widetilde{S_{0}}$. Thus the proof is completed.

\section{The proof of phase transition}

In this section, we will prove a phase transition result stated in Theorem 1.1 for the situation with $m=2$ and $f$ being the tent map. 
7.1 The case $0 \leq c<\frac{1}{4}$ (or $\frac{3}{4}<c \leq 1$ )

For this situation, $4|1-2 c|>2$ and there are four small squares on each of which the map is $1-1$ since $m=2$. It is easy to see that if a convex subset $\Omega_{0}$ of $[0,1]^{2}$ possesses nonempty intersections with at least three small cubes, then it must have nonempty intersection with the line $y=x$ or $x+y=1$. Then we can obtain the existence of a set of 'good' points occupying a fixed ratio $\left(\geq \epsilon^{2} / 2\right)$ in $\Omega_{0}$ by convexity (since all points in $\epsilon$-neighbor of the synchronization manifold are good and the diameter of $[0,1]^{2}$ is $\sqrt{2}$, see the argument in Lemma 6.1).

Hence we only need to consider the case that a convex subset $\Omega_{0}$ of $[0,1]^{2}$ possesses nonempty intersections with at most two small cubes. Obviously $\mathrm{M}\left(T\left(\Omega_{0}\right)\right) \geq 4 \mid 1-$ $2 c \mid \mathrm{M}\left(\Omega_{0}\right)$. Thus from Iteration Lemma 2.1 and Corollary 2.1 with $m_{0}=1, a=2$ and $E_{-}(c)=4|1-2 c|>2$, we complete the proof for the order part by the argument in section 6 (see Lemma 6.1 and Proposition 6.2).

For the disorder part, note that all the arguments in section 4 except Proposition 4.1 is available for each $c$ satisfying $\left|c-\frac{1}{2}\right|>\frac{1}{4}$. Thus we only need to provide a proof of Proposition 4.1 for $\left|c-\frac{1}{2}\right|>\frac{1}{4}$.

Recall that the proof of Proposition 4.1 depends on a claim that for a segment $\Omega \subset G_{\gamma_{0}}$ with a slope $\pm 1, T^{4}(\Omega)$ has at most two components. For small $c$, it holds that $(2(1-$ $2 c))^{4}>2$. Since the absolute value of each eigenvalue of $T$ is not less than $2(1-2 c)$, we have that each segment in $T^{4}(\Omega)$ possesses a length strictly longer than $(2(1-2 c))^{4} / 2$. $M(\Omega)$, where $M(\Omega)$ is the length of $\Omega$. It then implies Proposition 4.1 with the help of Iteration Lemma 2.1 and Corollary 2.1.

Since $(2(1-2 c))^{4}>2$ is true only for $c$ satisfying $\left|c-\frac{1}{2}\right|>2^{\frac{1}{4}} \cdot \frac{1}{4}$, it is sufficient to prove the following claim:

Claim'. Fix $c$ such that $\left|c-\frac{1}{2}\right|>\frac{1}{4}$. Let $M \in \mathbb{N}$ such that $(2(1-2 c))^{M}>2$. Then for sufficiently small $\gamma_{0}>0$ and a segment $\Omega$ in $G_{\gamma_{0}} \cap\left\{\right.$ some small square in $\left.[0,1]^{2}\right\}$ with a sufficiently small length such that $T^{l}(\Omega) \nsubseteq D_{\text {syn }}$ for any $l, T^{M}(\Omega)$ has at most two components.

The proof of it is same as the one for the Claim in section 4 . We omit it here. It is worthy to point out that $\gamma_{0}$ here depends on $c$. Thus we complete the proof for the disorder part.

7.2 The case $\frac{1}{4} \leq c \leq \frac{3}{4}$

For $\frac{1}{4}<c<\frac{3}{4}$, we have $2|1-2 c|<1$. Thus it is easy to see that

$$
\begin{aligned}
& \left|x_{1}(n+1)-x_{2}(n+1)\right| \leq 2|1-2 c|\left|x_{1}(n)-x_{2}(n)\right| \\
\leq & \cdots \leq(2|1-2 c|)^{n+1}\left|x_{1}(0)-x_{2}(0)\right| \rightarrow 0
\end{aligned}
$$

for any $\left(x_{1}(0), x_{2}(0)\right) \in[0,1]^{2}$. Hence synchronization occurs. 
When $c=\frac{1}{4}$ or $\frac{3}{4}$, by a direct computation, we know $\left|x_{1}(n+1)-x_{2}(n+1)\right|=$ $\left|x_{1}(n)-x_{2}(n)\right|$ for $\left(x_{1}(n)-\frac{1}{2}\right)\left(x_{2}(n)-\frac{1}{2}\right) \geq 0$, while $\left|x_{1}(n+1)-x_{2}(n+1)\right|<\mid x_{1}(n)-$ $x_{2}(n) \mid$ for $\left(x_{1}(n)-\frac{1}{2}\right)\left(x_{2}(n)-\frac{1}{2}\right)<0$. Hence $\left\{\left|x_{1}(n)-x_{2}(n)\right|\right\}$ is monotonically decreasing nonnegative sequence. It then has a limit. We need to prove the limit is zero for almost every initial data.

Otherwise, there is $d>0$ such that the limit $d_{\infty}>d$ for initial data occupying a positive measure. It then implies that for such an initial data,

$$
\left.\mid x_{1}(n)-x_{2}(n)\right) \mid>d
$$

for all sufficiently large $n$.

Suppose that

$$
\left|x_{1}(n)+x_{2}(n)-1\right| \leq \frac{d}{2} .
$$

It together with (7.1) implies that $\left(x_{1}(n)-\frac{1}{2}\right)\left(x_{2}(n)-\frac{1}{2}\right)<0$. Then again from (7.2) we have

$$
\begin{aligned}
& \left|x_{2}(n+1)-x_{1}(n+1)\right|=|1-2 c| \cdot\left|f\left(x_{2}(n)\right)-f\left(x_{1}(n)\right)\right| \\
= & |1-2 c| \cdot 2\left|1-x_{1}(n)-x_{2}(n)\right| \leq d|1-2 c|<d .
\end{aligned}
$$

It conflicts with (7.1).

Hence $\left|x_{1}(n)+x_{2}(n)-1\right|>\frac{d}{2}>0$ for all sufficiently large $n$. But then we have

$$
x_{2}(n+1)+x_{1}(n+1)= \begin{cases}2\left(x_{1}(n)+x_{2}(n)\right), & 0 \leq x_{1}(n), x_{1}(n) \leq \frac{1}{2} \\ 2\left(2-x_{1}(n)-x_{2}(n)\right), & \frac{1}{2} \leq x_{1}(n), \quad x_{1}(n) \leq 1\end{cases}
$$

It is equivalent to

$$
y(n+1)= \begin{cases}2 y_{1}(n), & 0 \leq y(n) \leq \frac{1}{2} \\ 2(1-y(n)), & \frac{1}{2} \leq y(n) \leq 1,\end{cases}
$$

where $y(n)=\left(x_{1}(n)+x_{2}(n)\right) / 2$ satisfying $\left|y(n)-\frac{1}{2}\right|>\frac{d}{4}>0$ for all sufficiently large $n$.

But it is impossible for almost every points from the dynamical behavior of the dynamical system $T: x \rightarrow 2 x \bmod 1$. Thus we obtain synchronization occurs for almost every initial data.

\section{Conclusions}

The clustering phenomenon intermittent behaviors have been widely found in coupled map lattices by numerical experiments but without mathematical proof. Among these 
phenomenon, pseudo synchronization, i.e., successive transition between ordered and disordered phases, is the most difficult from the point of view of mathematics. In this paper, we provide a complete proof for pseudo synchronization for weakly coupled tentmap lattices with arbitrarily many nodes. For weakly coupled piecewise-expanding map lattices with 2 nodes, we also obtain the same result. In particular, for coupled tent-map lattices with 2 nodes, we prove a phase transition between intermittent behaviors and synchronization.

How to extract more information on the dynamical properties by this work and previous results, for example, of G. Keller and C. Liverani [17-19], is one of our future interest. We will also be interested in the change of dynamical behavior when a strong coupling decreases to zero. In addition, we will study the weakly coupled piecewise-expanding map lattices with arbitrarily many nodes in the future.

\section{Acknowledgements}

We would like to thank G. Keller for his helpful comments. This work is supported by NSFC of China (Grants Nos. 11031003, 11271183, 11971105 and 11771205) and Simons Foundation.

\section{References}

[1] J. Bardet and G. Keller, Phase transitions in a piecewise expanding coupled map lattice with linear nearest neighbour coupling, Nonlinearity, 19(9) (2006), 2193-2211.

[2] M. Benedicks and L. Carleson, On iterations of $1-a x^{2}$ on (-1,1), Ann. Math., 122 (1985), $1-25$.

[3] S. Boccaletti, J. Kurths, G. Osipov, D. Valladares and C. Zhou, The synchronization of chaotic systems, Phys. Reports, 366(1-2) (2002), 1-101.

[4] C. Furusawa and K. Kaneko, Zipf's Law in Gene expression, Phys. Rev. Lett., 90(8) (2003), 088102.

[5] F. Giannakopoulos, U. Bihler, C. Hauptmann and H. Luhmann, Generation of epileptiform activity in a neocortical network: a mathematical model, Biol. Cybern., 85 (2001), 257-268.

[6] F. Giannakopoulos, C. Hauptmann and A. Zapp, Bursting activity in a model of a neuron with recurrent synaptic feedback, Fields Institute Communications, 29 (2001), 147-159.

[7] F. Hoppensteadt and E. Izhikevich, Weakly Connected Neural Networks, Springer, 1997, Applied Mathematical Sciences 126.

[8] E. Järvenpää, and M. Järvenpää, On the definition of SRB-measures for coupled map lattices, Commun. Math. Phys., 220 (2001), 1-12.

[9] K. Kaneko, Chaotic but regular posinega switch among coded attractors by cluster size variation, Phys. Rev. Lett., 63(3) (1989), 219-224.

[10] K. Kaneko, Clustering, coding, switching, hierarchical ordering, and control in network of chaotic elements, Phys. D, 41(2) (1990), 137-172.

[11] K. Kaneko, Globally coupled circle maps, Phys. D, 54 (1991), 5-19.

[12] K. Kaneko, Relevance of clustering to biological networks, Phys. D, 75 (1994), 55-73. 
[13] K. Kaneko, Coupled maps with growth and death: an approach to cell differentiation, Phys. D, 103 (1997), 505-527.

[14] K. Kaneko and T. Yomo, Cell divisions, differentiation and dynamical clustering, Phys. D, 75 (1994), 89-102.

[15] G. Keller, Mixing for finite systems of coupled tent maps, Tr. Mat. Inst. Steklova 216 (1997), Din. Sist. i Smezhnye Vopr., 320-326; translation in Proc. Steklov Inst. Math., (216) (1997), 315-332.

[16] G. Keller and M. Künzle, Transfer operators for coupled map lattices, Ergodic Theory Dynam. Syst., 12(2) (1992), 297-318.

[17] G. Keller and C. Liverani, A spectral gap for a one-dimensional lattice of coupled piecewise expanding interval maps, Dynamics of Coupled Map Lattices and of Related Spatially Extended Systems (Eds.: J.-R. Chazottes, B. Fernandez), Lecture Notes in Physics, 671 (2005), 115-151, Springer Verlag.

[18] G. Keller and C. Liverani, Uniqueness of the SRB measure for piecewise expanding weakly coupled map lattices in any dimension, Commun. Math. Phys., 262(1) (2006), 33-50.

[19] G. Keller and C. Liverani, Rare events, escape rates and quasistationarity: some exact formulae, J. Stat. Phys., 135(3) (2009), 519-534.

[20] T. Li and J. Yorke, Period 3 implies Chaos, Amer. Math. Monthly, 82 (1975), 985-992.

[21] C. Liverani, Decay of correlations for piecewise expanding maps, J. Stat. Phys., 78(3) (1995), 1111-1129.

[22] C. Liverani, Multidimensional expanding maps with singularities: a pedestrian approach, Ergodic Theory and Dynamical Systems, 33(1) (2013), 168-182.

[23] M. Lyubich, Almost every real quadratic map is either regular or stochastic, Ann. Math., 156(1) (2002), 1-78.

[24] L. Pecora, Synchronization conditions and desynchronizing patterns in coupled limit-cycle and chaotic systems, Phys. Rev. E, 58 (1998), 347-360.

[25] L. Pecora, T. Carroll, G. Johnson, D. Mar, and K. Fink, Synchronization stability in coupled oscillator arrays: Solution for arbitrary configurations, Int. J. Bifurc. Chaos, 10 (2000), 273290.

[26] B. Saussol, Absolutely continuous invariant measures for multidimensional expanding maps, Israel J. Math., 116 (2000), 223-248.

[27] L. Young, Developments in chaotic dynamics, AMS Notices, 45(10) (1998), 1318-1328. 